\title{
Inventory Model with Partial Backordering When Backordered Customers Delay Purchase after Stockout-Restoration
}

\author{
Ren-Qian Zhang, ${ }^{1}$ Yan-Liang Wu, ${ }^{1}$ Wei-Guo Fang, ${ }^{1}$ and Wen-Hui Zhou ${ }^{2}$ \\ ${ }^{1}$ School of Economics and Management, Beihang University, Beijing 100191, China \\ ${ }^{2}$ School of Business Administration, South China University of Technology, Guangzhou 510640, China \\ Correspondence should be addressed to Wen-Hui Zhou; whzhou@scut.edu.cn
}

Received 20 October 2015; Revised 11 January 2016; Accepted 14 January 2016

Academic Editor: Young Hae Lee

Copyright ( 2016 Ren-Qian Zhang et al. This is an open access article distributed under the Creative Commons Attribution License, which permits unrestricted use, distribution, and reproduction in any medium, provided the original work is properly cited.

\begin{abstract}
Many inventory models with partial backordering assume that the backordered demand must be filled instantly after stockout restoration. In practice, however, the backordered customers may successively revisit the store because of the purchase delay behavior, producing a limited backorder demand rate and resulting in an extra inventory holding cost. Hence, in this paper we formulate the inventory model with partial backordering considering the purchase delay of the backordered customers and assuming that the backorder demand rate is proportional to the remaining backordered demand. Particularly, we model the problem by introducing a new inventory cost component of holding the backordered items, which has not been considered in the existing models. We propose an algorithm with a two-layer structure based on Lipschitz Optimization (LO) to minimize the total inventory cost. Numerical experiments show that the proposed algorithm outperforms two benchmarks in both optimality and efficiency. We also observe that the earlier the backordered customer revisits the store, the smaller the inventory cost and the fill rate are, but the longer the order cycle is. In addition, if the backordered customers revisit the store without too much delay, the basic EOQ with partial backordering approximates our model very well.
\end{abstract}

\section{Introduction}

It is observed usually in daily life that customers who have incurred a stockout might not be avid to pay and fetch the backordered item definitely at the next replenishment point, when the stocked out item is available again. Consider that a customer visits an electronics store for a computer but incurs a stockout. If the customer is willing to backorder the demand, the manager will inform her/him of the date when the computer is available again. In an ideal situation, as assumed in the existing model of economic order quantity with partial backordering (EOQ-PBO), the customer will instantly revisit the store and fetch the backordered computer exactly at the next replenishment point $[1,2]$. It implies that the backordered customers generate an unlimited demand rate and the store need not keep any inventory for the backordered demand.

However, in some practical situations, it need take time to meet the backordered customer and the customer who is not in urgency is more possible to delay the purchase or decrease the order frequency to the store after incurring a stockout [3-5]. Thus, among the customers who have backordered the computer because of the stockout, some of them may repurchase the item immediately as soon as the inventory is replenished again, but some of them will revisit the store relatively later after the stockout-restoration, although they have incurred a stockout. Similarly, some other backordered customers may revisit the store more lately and so on.

Because of the purchase delay behavior, the backordered customers will repurchase the item generating a limited, not an unlimited, demand rate. Consequently, the vendor has to keep inventory for the backordered customers, resulting in a new cost component in the inventory model, which therefore leads to a different inventory policy for the vendor as a result of the different cost structure. This paper will reinvestigate the retailer-consumer inventory problem, considering that the backordered customers do not repurchase the item exactly at the next replenishment point but rather revisit the store 
successively during the next in-stock period because of the purchase delay behavior.

\section{Literature Review}

Assuming that a fraction of unsatisfied customers incurring stockouts is willing to backorder their demand and be satisfied by the next replenishment led to the problem of EOQ with partial backordering (EOQ-PBO) [1]. The basic EOQ-PBO model assumes that a constant percentage $\beta$ of the demand during the stockout period will be backordered, and the remaining fraction $1-\beta$ of the unsatisfied demand is lost [6-10]. Among different variants of the model [2], one of the most frequent extensions is to introduce a changing pattern for the backordering rate rather than assuming that it is a constant percentage [11-15]. These models have considered the psychology regarding how the backordered customers will wait the stockout-restoration before the next replenishment point, which is often varying with the waiting time.

Some researches considered new demand patterns rather than using the constant demand rate. The often adopted pattern is that demand is a time-varying function [16-22]. Another demand pattern adopted in EOQ-PBO is inventorylevel-dependent demand, which is also used in inventory model of perishable items [23-27]. Besides, some researches assumed that the demand rate was dependent on price and time $[28,29]$. We refer the reader to the survey of Pentico and Drake [2] for a more extensive survey. Recently, the problem was extended to consider prepayment, delayed payment, linear and fixed backordering costs, discounts, and so forth [30-33].

The above literature shows that most variants of the EOQPBO model are focused on different patterns of demand rate or backordering rate. The backordering rate describes how the unsatisfied customers will wait for the next replenishment. However, it does not consider the "sequel" after the customer completes the wait, that is, how the backordered customers revisit the store and fetch their backordered items after the next replenishment arrives and the item is available again.

Duran et al. [34] pointed out that unsatisfied customers may delay their purchase in the next replenishment period because they wish to obtain greater customer utility (either increased service or decreased price). More generally, Greenleaf and Lehmann [35] and G.-R. Chen and M.-L. Chen [36] reported that customers may delay their purchase just because they do not have enough time, feel unpleasant for shopping the product, experience perceived risk, rely on advice from others, wait for a lower price, and so forth. It is also possible that one may delay occasionally a purchase because of unexpected events [37]; for example, unexpected business meeting obstructs the backordered customer revisiting the store in time, or even the backordered customer delays the purchase due to avoidance and indecision [38]. Moore and Fitzsimons [39] further showed that consumers may respond negatively to the vendor after the stockoutrestoration. For online purchase situations, although retailers can promptly inform consumers of product availability, consumers do not immediately recover the negative effects of the stockout [40]. We can reasonably speculate that all these factors will considerably delay the repurchasing action of the backordered customers.

On the other hand, in some practical situations, the retailer may also delay delivering goods to the customers who have lower priorities [41]. Even if the backordered customers require that the vendor sends out the item as soon as it is available, they need still wait a number of days until the product is delivered [42]. In case of cash on delivery, it means that the repurchase is delayed and the retailer has to bear the inventory cost for keeping the backordered item on passage.

In sum, despite the possible delay of vendor or customer, an extra inventory of backordered demand should be held with longer time compared with the rigid assumption in most of the existing EOQ-PBO models that the backordered demand must be fulfilled completely as soon as the new order arrives. We have checked the above-mentioned literatures that are related to the customer's purchase delay behavior, but none of them had introduced the factor of purchase delay into inventory model [3-5, 34-41].

This paper extends the EOQ-PBO problem considering the purchase delay of the backordered customer; that is, the backordered demand will be fulfilled gradually during the subsequent replenishment period rather than being met outright at the very beginning of the contiguous inventory period. The key of the problem is to model the endogenous demand rate derived from the backordered customers and to formulate the inventory cost function considering new cost structure.

The rest of the paper is organized as follows. In Section 3, we present the problem definition and further list the new contributions of our study. Section 4 formulates the model by deriving the backorder demand rate and the total inventory cost function. Section 5 first briefly introduces Lipschitz Optimization (LO) used in the proposed algorithm. Subsequently, the internal-layer algorithm for obtaining the optimal order cycle with given fill rate is presented, based on which the external-layer algorithm for finding the globally optimal solution is proposed. Section 6 evaluates the performance of the proposed algorithm and presents managerial insights by numerical computations. Conclusions are given in Section 7.

\section{Problem Definition}

The traditional EOQ-PBO model assumes one constant demand rate, but the vendor in fact must handle two kinds of demand during each inventory period: (1) a stable demand for ordinary customers who have not incurred any stockouts (we call the corresponding demand per unit time routine demand rate); (2) an endogenous demand derived from the previousperiod backordered customers, which is characterized by the backorder demand rate.

Specifically, we assume that the endogenous demand is dependent on the amount of the backordered customers who have not been satisfied, which thereby generates a decreasing and convex backorder demand rate. Different from the existing inventory models, the inventory holding cost in this case will include two parts: the inventory holding cost of the item for meeting the routine demand and the 
TABLE 1: Cost components in related literature and our model.

\begin{tabular}{|c|c|c|c|}
\hline \multirow{2}{*}{ Inventory cost components/factors } & \multicolumn{2}{|c|}{ Papers reviewed } & \multirow{2}{*}{ Our model } \\
\hline & Stream I & Stream II & \\
\hline Ordering cost & $\sqrt{ }$ & $\times$ & $\sqrt{ }$ \\
\hline Inventory holding cost of in-stock items & $\sqrt{ }$ & $x$ & $\sqrt{ }$ \\
\hline Backorder cost of stockouts & $\sqrt{ }$ & $x$ & $\sqrt{ }$ \\
\hline Opportunity cost of lost sales & $\sqrt{ }$ & $x$ & $\sqrt{ }$ \\
\hline Repurchase delay of backordered customers & $x$ & $\sqrt{ }$ & $\sqrt{ }$ \\
\hline Describe/analyze the behavior of purchase delay & $x$ & $\sqrt{ }$ & $\sqrt{ }$ \\
\hline Endogenous demand rate of backordered customers & $x$ & $x$ & $\sqrt{ }$ \\
\hline Inventory holding cost of backordered demand & $\times$ & $\times$ & $\sqrt{ }$ \\
\hline Stream I & \multicolumn{3}{|c|}{$\begin{array}{l}\text { Montgomery et al. [1], Rosenberg [6], Park [7, 8], Wee [9], Padmanabhan and Vrat } \\
\text { [23], Wee [16], Abad [28, 29], Abboud and Sfairy [11], Skouri and Papachristos [17], } \\
\text { Zhou et al. [18], Ghosh and Chaudhuri [19], San José et al. [12-14], Hou [24], Jain et } \\
\text { al. [25], Uthayakumar and Geetha [26], Pentico and Drake [10], Shah [20], Yang et } \\
\text { al. [27], Dye and Hsieh [15], Taleizadeh et al. [30-32], and Wee et al. [33] }\end{array}$} \\
\hline Stream II & \multicolumn{3}{|c|}{$\begin{array}{l}\text { Ajzen [37], Greenleaf and Lehmann [35], Darpy [38], Zinn and Liu [3], Duran et al. } \\
\text { [34], Liberopoulos and Tsikis [4], Li and Chen [41], G.-R. Chen and M.-L. Chen } \\
\text { [36], Rao et al. [5], Pizzi and Scarpi [40], and Moore and Fitzsimons [39] }\end{array}$} \\
\hline
\end{tabular}

inventory holding cost of the item for satisfying the endogenous backordered demand. Moreover, considering ordering cost and the opportunity cost of lost sales, we will determine the optimal fill rate and the optimal replenishment interval so as to minimize the total inventory cost.

Our study is based on the literature regarding two streams. One is focused on the optimal inventory lot-sizing determined by minimizing the total inventory cost (Stream I in Table 1). The other is focused on customer purchasing behavior, referring mainly to the reason-explanation for delaying purchase and the related statistical analysis (Stream II in Table 1). Because of the endogenous backordered demand, the components of inventory cost are different from those of the existing inventory models. We compare our model with the existing models in Table 1, for clearly highlighting the new contribution of this paper.

To our knowledge, this is the first time that the backordered customer's purchase delay is introduced into the inventory model to minimize the total inventory cost. Model with this assumption is more complicate on account that we must handle with a new inventory cost component, that is, the inventory holding cost of the item kept for the backordered customers. What is more, a two-layer global optimization algorithm is needed for the solution because of the mathematical intractability.

\section{Formulation of the Model}

4.1. Notations and Assumptions. We keep all assumptions of the basic EOQ-PBO model $[2,10]$ except assuming that the backordered demand is filled instantly at the beginning of the very next order cycle with an infinitely large demand rate. If the backordered customers delay their repurchasing and therefore do not fetch the backordered items immediately at the beginning of the next order cycle, the corresponding demand rate is limited. Therefore, the store will incur an inventory holding cost of the backordered demand because of keeping inventory for the backordered customers, as in right side of Figure 1.

The notations in Notations section are used to model the problem.

Obviously, the total order quantity is equal to $D F T+\bar{B}$. It can be virtually divided into two parts: the first part DFT is used for satisfying the routine demand of the first time visiting (first-visiting) customers by the shelf stock during in-stock period. The corresponding demand rate is $D$. The second part, $\bar{B}=\beta D(1-F) T$, is ordered for satisfying the demand that is backordered during the previous stockout period and will be consumed according to the backorder demand rate $D_{b}(t)$. We can see from the left side of Figure 1 that if the backordered demand is satisfied instantly, then the inventory of the order quantity $\bar{B}$ for the backordered customers becomes 0 immediately as soon as the inventory is replenished, without causing any inventory holding cost; otherwise, the corresponding inventory level will gradually decrease to 0 and the store must bear an extra inventory holding cost (see the right side of Figure 1).

Different repurchasing behavior of backordered customers generates different backorder demand rate $D_{b}(t)$. Because of the complexity of the purchase delay factors, we consider an "average effect" of the purchase delay for facilitating modeling the inventory problem. That is, all the backordered customers are regarded as homogeneous so that at any time during the next in-stock period, each backordered customer has the same probability of revisiting the store and buying the backordered item. It means that during any unit time, the amount of the backordered customers who implement the repurchasing action should be proportional 


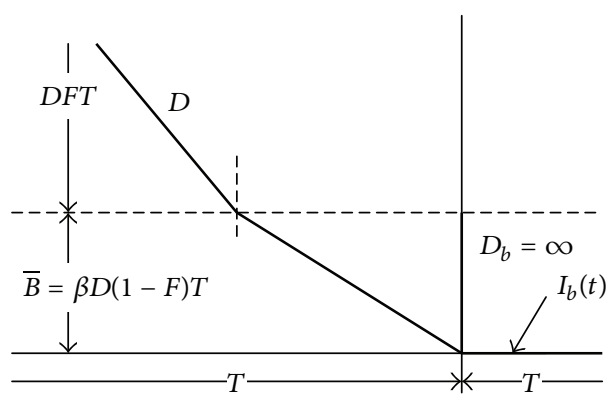

Backordered demand is satisfied instantly with unlimited demand rate $D_{b}=\infty$ (basic EOQ-PBO)

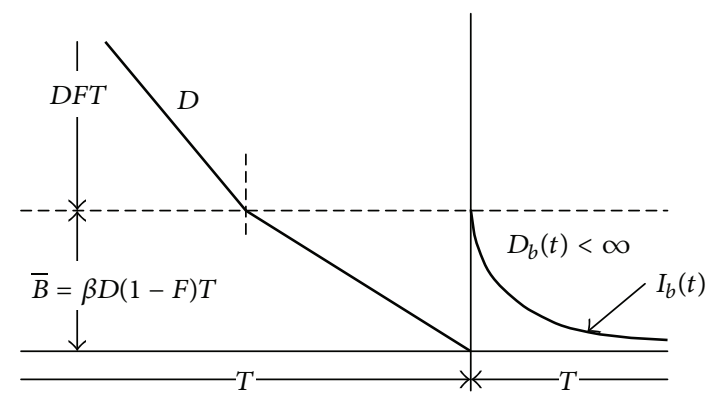

Backordered demand is satisfied gradually with a limited demand rate $D_{b}(t)$

FIgURE 1: Two different cases of satisfying the backordered demand.

to the remaining backordered customers, which is equally expressed in Assumption 1, as follows.

Assumption 1. The backorder demand rate is proportional to the remained unsatisfied backordered customers; that is, $D_{b}(t)=\alpha \mathscr{B}(t)$ and $\alpha$ is a positive real number.

Moreover, for the convenience in management, a vendor often commits one-time backorder for a backordered customer. It is possible that a so-called "backordered" customer might miss the entire very next in-stock period due to excessive purchase delay (the delay time is longer than $F T$ ). In this case, the customer will suffer a second stockout and it is highly possible that the corresponding demand will be lost forever due to customer's impatience [43, 44]. But in this case, we should not fault the vendor again for the "second-time" stockout, because it is completely caused by the customer's excessive purchase delay. Thus, this kind of "backordered" customers does not lead to any inventory cost and therefore does not impact the inventory model. In fact, if a "backordered" customer misses the next replenishment again and fades forever, the store should not replenish for the corresponding demand. This is equal to the case that the customer does not backorder the item at all when they incur the first time stockout. Thus, we can calibrate the backordering rate $\beta$ to eliminate the "backordered" customer who incurs a second stockout because of the excessive purchase delay time longer than $F T$. Hence, to facilitate modeling the inventory problem, we have Assumption 2.

Assumption 2. All the backordered customers must be completely satisfied during all the next in-stock period of $[0, F T]$.

It is also possible that all backordered customers are satisfied during a period of time that is shorter than FT. If it happens, our formulation will not precisely model the inventory problem. But we can approximate this case by adopting a very large attenuation coefficient, which ensures that almost all the backordered customers are satisfied before FT. From this point, we approximately assume, in Assumption 2, that the backordered customers are satisfied during all the instock period of $[0, F T]$.
4.2. The Backorder Demand Rate. Based on the backorder demand rate assumption (Assumption 1), we have a differential equation as

$$
D_{b}(t)=-\frac{d \mathscr{B}(t)}{d t}=\alpha \mathscr{B}(t), \quad \alpha>0 .
$$

The solution to the differential equation is $\mathscr{B}(t)=\mathscr{B}_{0} e^{-\alpha t}$, where $\mathscr{B}_{0}$ is a constant that is dependent on boundary conditions. Thus, the backorder demand rate at time $t$ is

$$
D_{b}(t)=-\frac{d \mathscr{B}(t)}{d t}=\alpha \mathscr{B}_{0} e^{-\alpha t} .
$$

The backorder demand rate $D_{b}(t)$ is an exponentially decreasing function in time $t$, where $\alpha$ in (2) is the attenuation coefficient of the backorder demand rate. $\alpha \mathscr{B}_{0}$ represents the initial demand rate at time $t=0$, which is dependent on how many demand has been backordered. We can see that a larger $\alpha$ corresponds to a larger backorder demand rate at the beginning of order cycle, but with a faster attenuation.

Since all the backordered customers are satisfied during the next in-stock period (Assumption 2), the total backordered demand $\bar{B}$ meets

$$
\begin{aligned}
\bar{B} & =\int_{t=0}^{F T} D_{b}(t) d t=\int_{t=0}^{F T} \alpha \mathscr{B}_{0} e^{-\alpha t} d t \\
& =\mathscr{B}_{0}\left(1-e^{-\alpha F T}\right) .
\end{aligned}
$$

The total quantity of backordered demand during each order cycle is $\bar{B}=\beta S=\beta D(1-F) T$ [10]. Therefore, $\mathscr{B}_{0}$ is determined by the following equation:

$$
\mathscr{B}_{0}=\frac{\beta D(1-F) T}{1-e^{-\alpha F T}} .
$$

\subsection{The Inventory Cost Function}

4.3.1. The Inventory Holding Cost of the Backordered Demand. Figure 2 presents the inventory level curves, where the upper part of the figure indicates how the backordered demands are satisfied in the coming order cycle and the lower part presents how the routine demand is satisfied. 


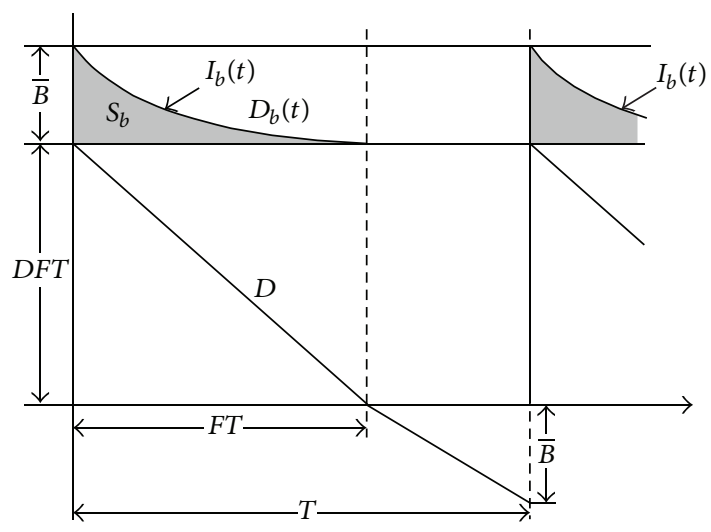

FIGURE 2: Inventory levels over time.

The backorder demand rate $D_{b}(t)$ exponentially attenuates and gradually reaches 0 , so that the backordered customers successively revisit the store during the in-stock period $[0, F T]$. Obviously, the inventory level corresponding to the backorder demand changes as

$$
\begin{aligned}
I_{b}(t) & =\bar{B}-\int_{\tau=0}^{t} D_{b}(\tau) d \tau=\bar{B}-\int_{\tau=0}^{t} \alpha \mathscr{B}_{0} e^{-\alpha \tau} d \tau \\
& =\mathscr{B}_{0}\left(e^{-\alpha t}-e^{-\alpha F T}\right) .
\end{aligned}
$$

Regarding the area under the inventory level curve of the backorder demand, we have

$$
S_{b}=\int_{t=0}^{F T} I_{b}(t) d t=\mathscr{B}_{0}\left[\frac{1}{\alpha}\left(1-e^{-\alpha F T}\right)-e^{-\alpha F T} F T\right] .
$$

Considering that $\mathscr{B}_{0}=\beta D(1-F) T /\left(1-e^{-\alpha F T}\right)$ (see (4)), the inventory holding cost per unit time of the backordered demand is

$$
\begin{aligned}
\frac{C_{h} S_{b}}{T} & =\frac{C_{h}}{T} \mathscr{B}_{0}\left[\frac{1}{\alpha}\left(1-e^{-\alpha F T}\right)-e^{-\alpha F T} F T\right] \\
& =\frac{\beta D C_{h}(1-F)}{\alpha}-\frac{\beta D C_{h}(1-F) F T}{e^{\alpha F T}-1} .
\end{aligned}
$$

4.3.2. The Total Inventory Cost Function. From the existing models, it is easy to know that the ordering cost of one unit time is $A / T$, the backordering cost per unit time is $\beta D C_{b}(1-F)^{2} T / 2$, the opportunity cost of lost sales is $C_{o} D(1-\beta)(1-F)$, and the holding cost of the inventory for satisfying the first-visiting customers is $D C_{h} F^{2} T / 2$. Summing up all the cost terms yields the total inventory cost per unit time as in the following:

$$
\begin{aligned}
\Gamma(T, F)= & \frac{A}{T}+\frac{D C_{h} F^{2}+\beta D C_{b}(1-F)^{2}}{2} T \\
& -\frac{\beta D C_{h}(1-F)}{\alpha} \frac{\alpha F T}{e^{\alpha F T}-1}+\frac{\beta D C_{h}(1-F)}{\alpha} \\
& +C_{o} D(1-\beta)(1-F) .
\end{aligned}
$$

Introduce auxiliary functions as follows:

$$
\begin{aligned}
& u(F)=\frac{\left[D C_{h} F^{2}+\beta D C_{b}(1-F)^{2}\right]}{2}, \\
& v(F)=\frac{\beta D C_{h}(1-F)}{\alpha}, \\
& w(F)=v(F)+C_{o} D(1-\beta)(1-F), \\
& \theta(x)=x\left(e^{x}-1\right)^{-1} .
\end{aligned}
$$

Then, the total cost function is recast as

$$
\Gamma(T, F)=\frac{A}{T}+u(F) T-v(F) \theta(x)+w(F),
$$

where $x=\alpha F T$. For the convenience, we denote $f^{\prime}(x)=$ $d f(x) / d x$ in this paper.

Compared with the existing EOQ-PBO model, the inventory total cost function of (10) has a new term of $\beta D C_{h}(1-$ $F) / \alpha-\left(\beta D C_{h}(1-F) / \alpha\right)\left(\alpha F T /\left(e^{\alpha F T}-1\right)\right)$. It leads to the intractability in finding the closed-form solution, which forces us to develop an algorithm based on Lipschitz Optimization (LO).

Note that the backordered customer may prepay a deposit for the backordered item. In this case, the store will incur less financial cost for the capital of inventory, where the unit inventory holding cost per unit time of the backordered item should be smaller than $C_{h}$, say $c_{h}<C_{h}$. To adapt the model for this case, we need only to substitute $c_{h}$ for $C_{h}$ in the auxiliary function $v(F)$ in the model. To be concise, we do not differentiate $c_{h}$ and $C_{h}$ in this paper.

\section{Solution}

\subsection{Solution Scheme and Lipschitz Optimization (LO)}

5.1.1. Solution Scheme. The convexity of the cost function $\Gamma(T, F)$ cannot be obtained and to derive a closed-form solution for the problem of $\min \Gamma(T, F)$ is very difficult, so we consider a two-layer strategy to find the optimal solution. Specifically, in the internal layer we minimize function $\Gamma(T, F)$ supposing that $F$ is given, say $F=\widetilde{F}$. This yields the solution to the problem of $\min _{T} \widetilde{\Gamma}(T, \widetilde{F})$ for any given $F=\widetilde{F} \in[0,1]$. Define a new function

$$
\widehat{\Gamma}(\widetilde{F})=\min _{T} \widetilde{\Gamma}(T, \widetilde{F}),
$$

representing the minimal value of $\Gamma(T, F)$ for $F=\widetilde{F}$.

In the external layer of the algorithm, we search the optimal value of $\widetilde{F}$ that minimizes function $\widehat{\Gamma}(\widetilde{F})$, which finally yields the globally optimal solution to the problem of $\min \Gamma(T, F)$. However, it is still very difficult to derive a closed-form solution even optimizing the single variable problem $\min _{T} \widetilde{\Gamma}(T, \widetilde{F})$ for $F=\widetilde{F}$, so we use Lipschitz Optimization (LO) to solve the problem of $\min _{T} \widetilde{\Gamma}(T, \widetilde{F})$ and $\min _{\widetilde{F}} \widehat{\Gamma}(\widetilde{F})$, respectively.

Function $f(x)$ is Lipschitzian on interval $[a, b]$ if, for any two points $x, y \in[a, b]$, the absolute difference of the 


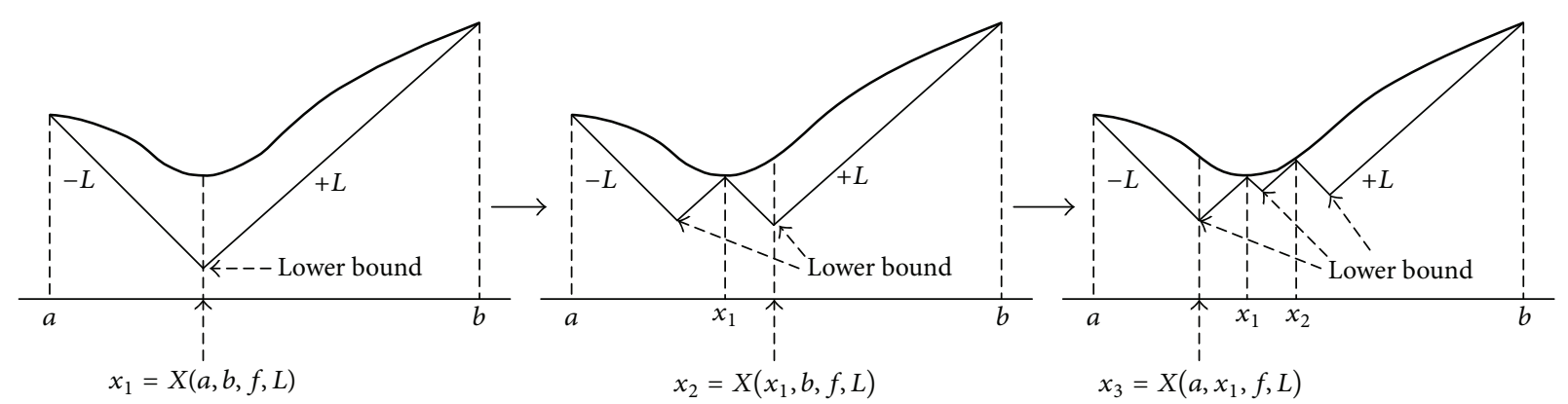

FIgURE 3: The basic idea of Shubert's algorithm.

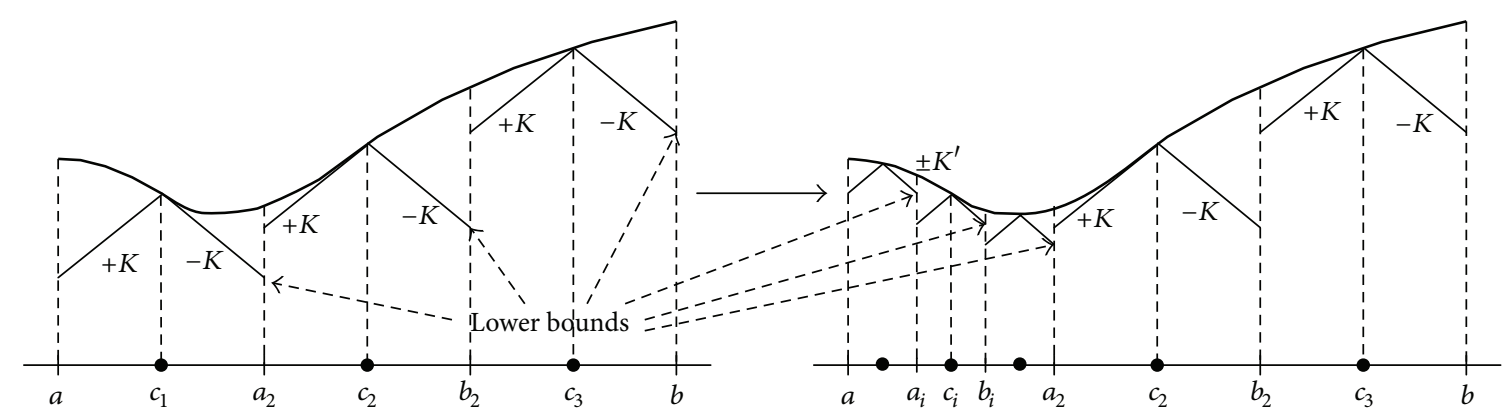

FIGURE 4: Interval partition of the DIRECT algorithm.

function is bounded by a constant multiplying the distance between $x$ and $y$. That is, $|f(x)-f(y)| \leq L|x-y|$, where $L$ is the Lipschitz constant. We have found that it is easy to determine the Lipschitz constant for function $\widetilde{\Gamma}(T, \widetilde{F})$ given $F=\widetilde{F}$, and therefore the LO algorithm with known Lipschitz constant can be applied to optimizing function $\widetilde{\Gamma}(T, \widetilde{F})$. However, the Lipschitz constant of function $\widehat{\Gamma}(\widetilde{F})$ is very difficult to be obtained, so we have to optimize function $\widehat{\Gamma}(\widetilde{F})$ without knowing Lipschitz constant. Below, we briefly introduce the basic idea of LO algorithms and the reader is referred to the related literature for the details $[45,46]$.

5.1.2. Shubert's Algorithm with a Known Lipschitz Constant. There are several LO algorithms for optimizing a Lipschitz function if the Lipschitz constant $L$ is known. The passive algorithm is the simplest one that evaluates the function on points $a+\varepsilon / L, a+3 \varepsilon / L, a+5 \varepsilon / L, \ldots \in[a, b]$, where the function value on the best point does not differ more than $\varepsilon$ from the global optimal value.

A more efficient LO method is Shubert's algorithm. Shubert's algorithm uses piecewise linear functions with slopes of $L$ and $-L$ to approximate the objective function from below on interval $[a, b]$ (see Figure 3 ).

Based on the piecewise linear function approximation, the algorithm continuously selects the interval with the minimal lower bound and partitions it into subintervals until optima are obtained under a given preciseness. We refer the reader to Shubert [45] for the details. In this paper, we will adopt Shubert's algorithm to minimize $\widetilde{\Gamma}(T, \widetilde{F})$, obtaining the optimal value of $T$ with given $F$.
5.1.3. The DIRECT Algorithm without Lipschitz Constant. Jones et al. [46] proposed a new variant of LO algorithm, named DIRECT, which does not need to know the Lipschitz constant. It is different from Shubert's algorithm that the DIRECT algorithm approximates the objective function from the middle point of the interval and partitions the selected interval (the interval with minimal lower bound) into three equal parts, as shown in Figure 4.

Figure 4 shows that we first partition the interval into three equal parts whose centers are $c_{1}, c_{2}$, and $c_{3}$, respectively. The two lines crossing the middle points $c_{1}, c_{2}$, and $c_{3}$ with slopes $\pm K$ provide lower bounds for the objective function on intervals $\left[a, a_{2}\right],\left[a_{2}, b_{2}\right]$, and $\left[b_{2}, b\right]$, respectively. Then select the interval with minimal lower bound, that is, $\left[a, a_{2}\right]$, for the further trisection while updating $K$ with $K^{\prime}$.

Generally, let $c_{i}=\left(a_{i}+b_{i}\right) / 2$ denote the center of interval $\left[a_{i}, b_{i}\right]$. The two lines crossing the middle point $c_{i}$ with slopes $\pm K$ provide lower bound for the objective function on interval $\left[a_{i}, b_{i}\right]$. Denote $\bar{c}_{i}=b_{i}-a_{i}$, the width of interval $\left[a_{i}, b_{i}\right]$. It is noticeable that there are multiple intervals corresponding to each value of $\bar{c}_{i}$. Each pair of $\bar{c}_{i}$ and $f\left(c_{i}\right)$ corresponds to a point with coordinates $\left(\bar{c}_{i}, f\left(c_{i}\right)\right)$ on a two-dimensional plane. As long as DIRECT selects the interval for the further trisection, the value of $K$ can be updated for the new iteration by finding the lower right of the convex hull of the set of points $\left(\bar{c}_{i}, f\left(c_{i}\right)\right)$, which can be realized by the conhull algorithm proposed in Björkman and Holmström [47]. The above procedure is repeated until the convergence basin of the optimum is found. Obviously, the DIRECT algorithm continuously renews $K$ to determine the slope of the crossing lines for approximating the objective 
function, so the Lipschitz constant is not needed. To save space, we refer the reader to Jones et al. [46] for the details of the DIRECT algorithm.

\subsection{The Optimal Value of T When F Is Given}

5.2.1. The First-Order Condition of Optimality. If $F$ is given, say $F=\widetilde{F}$, we introduce two functions $r(T)$ and $q(T)$ as follows:

$$
\begin{aligned}
& r(T)=\frac{A}{T}+u(\widetilde{F}) T, \\
& q(T)=v(\widetilde{F}) \theta(\alpha \widetilde{F} T) .
\end{aligned}
$$

Then, the cost function with given $F$ is recast as

$$
\widetilde{\Gamma}(T)=\widetilde{\Gamma}(T, \widetilde{F})=r(T)-q(T)+w(\widetilde{F}) .
$$

The optimal value of $\widetilde{\Gamma}(T, \widetilde{F})$ defined as $\widehat{\Gamma}(\widetilde{F})=\min _{T} \widetilde{\Gamma}(T, \widetilde{F})$ in (11) is

$$
\widehat{\Gamma}(\widetilde{F})=\min _{T} \widetilde{\Gamma}(T, \widetilde{F})=\min _{T} \widetilde{\Gamma}(T)
$$

Proposition 3 gives the first-order necessary condition for the minima of function $\widetilde{\Gamma}(T)$.

Proposition 3. The minima of function $\widetilde{\Gamma}(T)$ solve the following equation:

$$
\widetilde{\Gamma}^{\prime}(T)=r^{\prime}(T)-q^{\prime}(T)=0,
$$

where

$$
\begin{aligned}
& r^{\prime}(T)=-\frac{A}{T^{2}}+u(\widetilde{F}), \\
& q^{\prime}(T)=\alpha \widetilde{F} \cdot v(\widetilde{F}) \cdot \theta^{\prime}(\alpha \widetilde{F} T) .
\end{aligned}
$$

Proof. When $T \rightarrow 0, r(T) \rightarrow \infty$ and $q(T)>0$, so $\widetilde{\Gamma}(T) \rightarrow$ $\infty$. On the other hand, if $T \rightarrow \infty$, we also have $\widetilde{\Gamma}(T) \rightarrow$ $\infty$. Moreover, $\widetilde{\Gamma}(T)$ is continuous and differentiable, and for a given $T, \widetilde{\Gamma}(T)$ has a finite value. Hence, the minima of function $\widetilde{\Gamma}(T)$ meet the first-order condition. This completes the proof.

5.2.2. Bounds of the Optimal Value of T with Given F. Denote $\widehat{T}_{F}$ the optimal value of $T$ with given $F$. But even if $F$ is known, the convexity of function $\widetilde{\Gamma}(T)$ cannot be obtained, and it is also difficult to solve the first-order necessary condition to obtain $\widehat{T}_{F}$ for minimizing $\widetilde{\Gamma}(T)$. To use LO to find the optimal solution for a given $F$, we first determine the bounds of $\widehat{T}_{F}$.

Proposition 4. Let $\bar{T}_{F}$ denote the upper bound of $\widehat{T}_{F}$ and define a sequence $\left\{\bar{T}_{F}^{(k)}\right\}$ as

$$
\bar{T}_{F}^{(k)}=\sqrt{\frac{A}{u(\widetilde{F})-\alpha \widetilde{F} \cdot v(\widetilde{F}) \cdot \theta^{\prime}\left(\bar{x}^{(k-1)}\right)}},
$$

where $\bar{x}^{(k-1)}=\alpha \widetilde{F} \bar{T}_{F}^{(k-1)},(k=1, \ldots, \infty)$, and $\bar{T}_{F}^{(0)}=$ $\sqrt{A / u(\widetilde{F})}$. Then, $\widehat{T}_{F} \leq \bar{T}_{F}^{(k)}$ such that, for any $k, \bar{T}_{F}^{(k)}$ provides an upper bound for $\widehat{T}_{F}$ and $\bar{T}_{F}=\lim _{k \rightarrow \infty} \bar{T}_{F}^{(k)}$ is the minimal upper bound of $\widehat{T}_{F}$.

Proof. See Appendix B.

Proposition 5. Let $\underline{T}_{F}$ denote the lower bound of $\widehat{T}_{F}$ and define a sequence $\left\{\underline{T}_{F}^{(k)}\right\}$ as

$$
\underline{T}_{F}^{(k)}=\sqrt{\frac{A}{u(\widetilde{F})-\alpha \widetilde{F} \cdot v(\widetilde{F}) \cdot \theta^{\prime}\left(\underline{x}^{(k-1)}\right)},}
$$

where $\underline{x}^{(k-1)}=\alpha \widetilde{F} \underline{T}_{F}^{(k-1)},(k=1, \ldots, \infty)$, and $\underline{T}_{F}^{(0)}=$ $\sqrt{A /(u(\widetilde{F})+\alpha \widetilde{F} \cdot v(\widetilde{F}) / 2)}$. Then, $\widehat{T}_{F} \geq \underline{T}_{F}^{(k)}$ such that, for any $k, \underline{T}_{F}^{(k)}$ provides a lower bound for $\widehat{T}_{F}$ and $\underline{T}_{F}=\lim _{k \rightarrow \infty} \underline{T}_{F}^{(k)}$ is the maximal lower bound of $\widehat{T}_{F}$.

Proof. See Appendix B.

Propositions 4 and 5 give the bounds of $\widehat{T}_{F}$. For the limits of upper and lower bounds when $k \rightarrow \infty$, we have Proposition 6.

Proposition 6. The limits of upper and lower bounds both solve the first-order necessary condition given by (15).

Proof. See Appendix B.

Corollary 7. If $\alpha \rightarrow \infty$, that is, the backorder demand rate is infinitely large, the upper and lower bounds are identical and both solve the first-order condition of the basic EOQ-PBO model.

Proof. It is easy to verify that $\lim _{x \rightarrow \infty} \theta^{\prime}(x)=0$ (see Lemma A.2 in Appendix A). Thus, when $\alpha \rightarrow \infty$, we have $x=\alpha F T \rightarrow \infty$ and so $\lim _{k \rightarrow \infty} \bar{T}_{F}^{(k)}=\lim _{k \rightarrow \infty} \underline{T}_{F}^{(k)}=$ $\sqrt{2 A / u(F)}=\sqrt{2 A / D\left[C_{h} F^{2}+\beta C_{b}(1-F)^{2}\right]}$. This is equation (9) of Pentico and Drake [10], which solves the first-order condition of the basic EOQ-PBO model. The proof is completed.

Corollary 7 shows that the basic EOQ-PBO is a special case of our model. If $\alpha$ is sufficiently large, we can use the basic EOQ-PBO model as an approximation. The numerical experiment in Section 6 suggests that if $\alpha>30$, the basic EOQ-PBO can approximate our model very well.

Corollary 8. For $\alpha<\infty$, if the limits of upper and lower bounds are identical, that is, $\bar{T}_{F}=\underline{T}_{F}$, then they are the unique optimal solution to the problem of $\min _{T} \widetilde{\Gamma}(T)$; that is, $\widehat{T}_{F}=\bar{T}_{F}=\underline{T}_{F}$.

Proof. It is straightforward from Propositions 3 and 6.

Corollary 8 allows us to improve the efficiency of finding the optimal solution $\widehat{T}_{F}$. For a given $F$, we first check if the 
limits of the upper and lower bounds are identical. Only when the two limits are not equal, should we further use some optimization algorithms, such as the LO algorithm, to minimize function $\widetilde{\Gamma}(T)$; otherwise, the optimal value $\widehat{T}_{F}=$ $\bar{T}_{F}=\underline{T}_{F}$ is attained. Proposition 9 presents a sufficient condition under which $\bar{T}_{F}$ and $\underline{T}_{F}$ are equal to each other.

Proposition 9. The optimal value of order cycle $T$ with given $F$ can be determined by $\widehat{T}_{F}=\bar{T}_{F}=\underline{T}_{F}$ if the following inequality holds:

$$
\alpha^{2} \widetilde{F}^{2} v(\widetilde{F}) \sqrt{A}<12\left[u(\widetilde{F})-\alpha \widetilde{F} v(\widetilde{F}) \theta^{\prime}\left(\alpha \widetilde{F} \bar{T}_{F}\right)\right]^{3 / 2} .
$$

Proof. See Appendix B.

5.2.3. Lipschitz Optimization on $T$ with Given F. Based on the bounds of $T$ determined by Propositions 4 and 5, we can use LO to minimize $\widetilde{\Gamma}(T)$ when $F$ is given, for which Proposition 10 presents the Lipschitz constant.

Proposition 10. For $T_{1}, T_{2} \in\left[\underline{T}_{F}, \bar{T}_{F}\right]$, one has $\mid \widetilde{\Gamma}\left(T_{1}\right)-$ $\widetilde{\Gamma}\left(T_{2}\right)\left|\leq L_{\widetilde{F}} \cdot\right| T_{1}-T_{2} \mid$, where $L_{\widetilde{F}}$ is the Lipschitz constant that is determined by

$$
\begin{gathered}
L_{\widetilde{F}}=\max \left\{\left|u(\widetilde{F})+\frac{\alpha \widetilde{F} \cdot v(\widetilde{F})}{2}-\frac{A}{\bar{T}_{F}^{2}}\right|,\right. \\
\left.\left|u(\widetilde{F})-\frac{A}{\underline{T}_{F}^{2}}\right|\right\} .
\end{gathered}
$$

Proof. See Appendix B.

Additionally, Proposition 9 shows that if the limits of the upper and lower bounds are identical, then the limit directly gives the optimal value of $T$ as $\widehat{T}_{F}=\bar{T}_{F}=\underline{T}_{F}$. In this case, we do not need to use the LO method. The procedure of the algorithm optimizing $T$ with given $F$ is detailed as follows. We name this algorithm Opt_Local for the convenience.

\section{Algorithm Opt_Local}

Step 1. For given $F=\widetilde{F}$, calculate the limit of the upper bound $\bar{T}_{F}$ from (17), by iterations.

Step 2. Check whether (19) holds. If yes, the optimal value of $T$ with given $F$ is obtained by $\widehat{T}_{F}=\bar{T}_{F}$ and go to Step 6; otherwise, go to Step 3.

Step 3. Calculate the limit of the lower bound $\underline{T}_{F}$ from (18) by iterations.

Step 4. If $\underline{T}_{F}=\bar{T}_{F}$, then the optimal value of $T$ with given $F$ is obtained by $\widehat{T}_{F}=\underline{T}_{F}=\bar{T}_{F}$ and go to Step 6; otherwise, go to Step 5.

Step 5. Determine the Lipschitz constant from (20). Apply Lipschitz Optimization (Shubert's algorithm) to the problem of $\min _{T} \widetilde{\Gamma}(T)$ and the optimal value $\widehat{T}_{F}$ is obtained.
Step 6. Output the optimal solution $\widehat{T}_{F}$, and $\widehat{\Gamma}(\widetilde{F})=$ $\min _{T} \widetilde{\Gamma}(T)=\widetilde{\Gamma}\left(\widehat{T}_{F}\right)$.

In Step 5, we set the stop criterion based on relative improvements. Specifically, if the relative improvement on the objective function $\widetilde{\Gamma}(T)$ between two successive iterations keeps smaller than a very small positive number $\varepsilon$ for $p$ consecutive iterations, then stop. In this paper, we set $\varepsilon=10^{-6}$ and $p=10$. Thus, if the relative improvement keeps smaller than $0.0001 / \%$ for 10 consecutive iterations, stop.

5.3. Algorithm for Finding the Globally Optimal Solution. To obtain the global minima, we need minimize function $\widehat{\Gamma}(\widetilde{F})=\min _{T} \widetilde{\Gamma}(T, \widetilde{F})$ to find the optimal fill rate $F$. The convexity of $\widehat{\Gamma}(\widetilde{F})$ cannot be obtained, so the LO algorithm is employed again. Unfortunately, it is very difficult to derive closed-form expression for the Lipschitz constant of $\widehat{\Gamma}(\widetilde{F})$, so we adopt the DIRECT algorithm that does not need the Lipschitz constant. The condition of using DIRECT is that the objective function should at least be continuous within the neighborhood of global optima [46].

Consider two values of $F$, say $F=\widetilde{F}$ and $F=\widetilde{F}^{\prime}$. As mentioned above, with given $F$, the inventory cost function $\Gamma(T, F)$ is optimized by algorithm Opt_Local, yielding $\widehat{\Gamma}(\widetilde{F})=$ $\Gamma\left(\widehat{T}_{\widetilde{F}}, \widetilde{F}\right)$ and $\widehat{\Gamma}\left(\widetilde{F}^{\prime}\right)=\Gamma\left(\widehat{T}_{\widetilde{F}^{\prime}}, \widetilde{F}^{\prime}\right)$, respectively. Since function $\Gamma(T, F)$ is differentiable and continuous in $(T, F)$, if the difference between $\widetilde{F}^{\prime}$ and $\widetilde{F}$ is small enough, $\widehat{\Gamma}\left(\widetilde{F}^{\prime}\right)$ can be sufficiently close to $\widehat{\Gamma}(\widetilde{F})$; that is, $\lim _{\widetilde{F}^{\prime} \rightarrow \widetilde{F}} \widehat{\Gamma}\left(\widetilde{F}^{\prime}\right)=\widehat{\Gamma}(\widetilde{F})$. Therefore, $\widehat{\Gamma}(\widetilde{F})$ is continuous in $\widetilde{F}$.

The one-dimensional DIRECT algorithm optimizing function $\widehat{\Gamma}(\widetilde{F})$, named Algorithm Opt_Global, is depicted as follows.

\section{Algorithm Opt_Global}

Step 1. Divide the selected intervals within $[0,1]$ by the DIRECT algorithm into three equal sections and yield candidate points of $\widetilde{F} \in[0,1]$.

Step 2. Determine the value of $\widehat{\Gamma}(\widetilde{F})$ on each candidate point of $\widetilde{F}$ by using Algorithm Opt_Local and obtain the current best solution.

Step 3. Check whether the stop criterion is met. If yes, go to Step 5; otherwise, go to Step 4.

Step 4. Update $K$, the slope of the crossing linear functions, by calling the algorithm of conhull [47], and approximate the lower bound of function $\widehat{\Gamma}(\widetilde{F})$ for each interval by using the crossing linear function with slope $K$. Intervals with the smallest lower bound of function $\widehat{\Gamma}(\widetilde{F})$ are selected for the further trisection. Go to Step 1.

Step 5. Output the globally optimal solution.

Note that, in the first iteration, after partitioning the original interval $[0,1]$ into three equal subintervals, we select 
the subinterval for the next trisection directly based on the function value of the middle point of each subinterval, without approximating the objective function by the crossing lines with slope $K$. $K$ will be obtained and updated starting from the second iteration. Moreover, if the relative improvement on the objective function between two successive iterations keeps smaller than $\varepsilon=10^{-6}$ for $p=10$ consecutive iterations, then stop. In addition, as Zhang [48] showed that we must further compare $\Gamma^{*}$, the optimal value of $\Gamma(T, F)$, with $C_{o} D$, the opportunity cost of not stocking at all, to determine the optimal inventory policy. The smaller one is the optimal decision.

\section{Computational Study}

6.1. Benchmark Algorithms. To evaluate the performance of Algorithm Opt_Global, we use two algorithms as benchmarks. The first one is a grid search (GS) algorithm for evaluating the optimality of Algorithm Opt_Global, and the second one is a two-dimensional DIRECT algorithm [46] for evaluating the computational efficiency.

6.1.1. Grid Search on $\widetilde{F}$. We use grid search (GS) to approximately optimize function $\widehat{\Gamma}(\widetilde{F})$ and compare the solution with that obtained by Algorithm Opt_Global. For implementing the GS algorithm, we divide the interval $\widetilde{F} \in[0,1]$ by steps of $10^{-4}$. For each problem instance, we need to optimize function $\widetilde{\Gamma}(T)=\widetilde{\Gamma}(T, \widetilde{F})$ for ten thousand times by calling Algorithm Opt_Local and keep the value of $\widetilde{F}$ with minimal inventory cost as the optimal solution.

6.1.2. Two-Dimensional DIRECT Algorithm. The problem can also be solved by using the two-dimensional DIRECT algorithm (TD) proposed by Jones et al. [46]. However, the two-dimensional split of the space can be very timeconsuming if requiring a high precision. We here compare the performance of the Opt_Global algorithm with that of the TD algorithm to show how much Opt_Global will reduce the computational effort. The stop condition of the TD algorithm is the same as that of Algorithm Opt_Global; that is, the relative improvement keeps smaller than $10^{-6}$ for 10 consecutive iterations.

6.2. Outline of Numerical Examples. We investigate the performance of our model and algorithm under different scenarios by solving numerical examples. The parameter of the numerical examples should cover different cases as many as possible. Thus, we enumerate different values of parameters to construct different examples, referring to the parameter scheme of the major item of the numerical examples in Zhang et al. [49], as follows.

(i) Ordering Cost. We consider four values, $A=\$ 100 /$ order, $\$ 1000$ /order, $\$ 2500 /$ order, and \$5000/order, respectively, representing the difference in lower and higher ordering cost.

(ii) Inventory Holding Cost and Backordering Cost. Set $C_{h}=$ \$5/unit/year, \$10/unit/year, \$25/unit/year, and \$50/unit/year representing the inventory holding cost changes from low to high. Similarly, we set backorder cost as $C_{b}=\$ 5 /$ unit/year, $\$ 10 /$ unit/year, \$25/unit/year, or \$50/unit/year, respectively.

(iii) Opportunity Cost. Set $C_{o}=\$ 5 /$ unit, $\$ 10 /$ unit, $\$ 25 /$ unit, and $\$ 50 /$ unit, respectively.

(iv) Backordering Rate. Backordering rate $\beta$ is changed from 0.1 to 0.9 with a step of 0.2 .

(v) Demand. Set the routine demand rate $D=100$ units/year, 1000 units/year, 5000 units/year, and 10000 units/year, respectively.

(vi) Attenuation Coefficient of the Backorder Demand Rate. Set $\alpha=0.1,0.5,1,5,10,50,100,500$, representing the backorder demand rate attenuates from slow to fast.

By combination of $A \times C_{h} \times C_{b} \times C_{o} \times \beta \times D \times \alpha=4 \times$ $4 \times 4 \times 4 \times 5 \times 4 \times 8$ we generate 40960 different numerical examples for computational experiments.

6.3. Performance of the Algorithm. We apply, respectively, the three algorithms: Algorithm Opt_Global, grid search (GS), and the two-dimensional DIRECT (TD) algorithm to solve the generated 40960 numerical examples. Let $\Gamma_{\mathrm{Opt}}, \Gamma_{\mathrm{GS}}$, and $\Gamma_{\mathrm{TD}}$ denote the best value of the objective function obtained by Opt_Global, GS, and TD, respectively. Let $\left(\Gamma_{\mathrm{GS}}-\Gamma_{\mathrm{Opt}}\right) / \Gamma_{\mathrm{Opt}}$ indicate the relative deviation of the solution found by GS from that obtained by the Opt_Global algorithm, and $\left(\Gamma_{\mathrm{TD}}-\right.$ $\left.\Gamma_{\text {Opt }}\right) / \Gamma_{\text {Opt }}$ indicate the relative deviation of TD. We group all the numerical examples by the attenuation coefficient $\alpha$, and the computational results are presented in Table 2.

It can be observed from Table 2 that Algorithm Opt_Global performs better than grid search (GS) and twodimensional DIRECT (TD). This verifies the optimality of Opt_Global. Moreover, if we naively apply TD to solve the problem, it yields the worst solution among the three algorithms.

We also evaluate the computational efficiency through the computational time (on a PC with $3.4 \mathrm{GHz}$ Intel CPU and 4 GB memory), which is presented in Table 3.

Table 3 shows that the Opt_Global algorithm is much more efficient than GS and TD, in terms of computational time of CPU. In particular, TD may consume more than one hour to get the solution for some cases but obtain inferior results compared with that of Opt_Global (Table 2). Thus, it is reasonable to conclude that the two-layer structure in the proposed algorithm significantly improves the computational efficiency.

Note that we have tried searching the optimal value of $F$ by smaller steps of $10^{-6}$ to improve the preciseness of the GS algorithm, but it cannot improve the solution significantly. What is more, under the search step of $10^{-6}$, it costs more than 4000 seconds for the computer to solve one example by GS and we totally need more than three years to complete the computation of all the numerical examples on our computer. Thus, a search step of $10^{-4}$ for $F$ is used, with which all the numerical examples were solved within 20 days by the GS algorithm. 
TABLE 2: Relative deviations of the two benchmarks.

\begin{tabular}{|c|c|c|c|c|c|c|}
\hline \multirow{2}{*}{$\alpha$} & \multicolumn{3}{|c|}{ Dev. of $\Gamma_{\mathrm{GS}}(\%)$} & \multicolumn{3}{|c|}{ Dev. of $\Gamma_{\mathrm{TD}}(\%)$} \\
\hline & Min. & Avg. & Max. & Min. & Avg. & Max. \\
\hline 0.1 & $<10^{-6}$ & $8.03 \times 10^{-6}$ & $2.06 \times 10^{-2}$ & $<10^{-6}$ & $2.43 \times 10^{-5}$ & $6.16 \times 10^{-1}$ \\
\hline 0.5 & $<10^{-6}$ & $3.57 \times 10^{-4}$ & $3.88 \times 10^{-2}$ & $<10^{-6}$ & $3.25 \times 10^{-4}$ & $2.15 \times 10^{-1}$ \\
\hline 1 & $<10^{-6}$ & $5.05 \times 10^{-4}$ & $3.88 \times 10^{-2}$ & $<10^{-6}$ & $2.22 \times 10^{-4}$ & $4.21 \times 10^{-3}$ \\
\hline 5 & $<10^{-6}$ & $7.69 \times 10^{-4}$ & $3.88 \times 10^{-2}$ & $<10^{-6}$ & $4.46 \times 10^{-4}$ & $6.95 \times 10^{-1}$ \\
\hline 10 & $<10^{-6}$ & $2.81 \times 10^{-4}$ & $2.56 \times 10^{-2}$ & $<10^{-6}$ & $2.42 \times 10^{-4}$ & $4.22 \times 10^{-1}$ \\
\hline 50 & $<10^{-6}$ & $4.58 \times 10^{-5}$ & $2.02 \times 10^{-2}$ & $<10^{-6}$ & $3.51 \times 10^{-4}$ & $3.85 \times 10^{-1}$ \\
\hline 100 & $<10^{-6}$ & $4.32 \times 10^{-5}$ & $3.90 \times 10^{-2}$ & $<10^{-6}$ & $4.13 \times 10^{-4}$ & $9.12 \times 10^{-2}$ \\
\hline 500 & $<10^{-6}$ & $6.35 \times 10^{-5}$ & $1.72 \times 10^{-2}$ & $<10^{-6}$ & $2.70 \times 10^{-5}$ & $5.13 \times 10^{-1}$ \\
\hline
\end{tabular}

TABLE 3: Computational times of the three algorithms.

\begin{tabular}{|c|c|c|c|c|c|c|c|c|c|}
\hline \multirow{2}{*}{$\alpha$} & \multicolumn{3}{|c|}{ CPU time of Opt_Global (s) } & \multicolumn{3}{|c|}{ CPU time of GS (s) } & \multicolumn{3}{|c|}{ CPU time of TD (s) } \\
\hline & Min. & Avg. & Max. & Min. & Avg. & Max. & Min. & Avg. & Max. \\
\hline 0.1 & 0.41 & 1.90 & 2.85 & 41.14 & 44.41 & 53.01 & 2.902 & 17.92 & 4769 \\
\hline 0.5 & 0.40 & 1.68 & 2.74 & 42.76 & 44.26 & 53.13 & 3.058 & 18.56 & 4957 \\
\hline 1 & 0.41 & 1.92 & 3.14 & 42.38 & 44.19 & 53.27 & 3.125 & 18.66 & 5023 \\
\hline 5 & 0.41 & 1.92 & 3.75 & 42.15 & 44.12 & 53.42 & 3.117 & 19.22 & 5129 \\
\hline 10 & 0.39 & 1.87 & 4.00 & 42.19 & 42.50 & 53.85 & 3.211 & 17.84 & 4883 \\
\hline 50 & 0.39 & 1.59 & 4.26 & 42.70 & 42.92 & 47.24 & 4.259 & 19.56 & 5212 \\
\hline 100 & 0.25 & 1.83 & 4.19 & 42.23 & 42.89 & 52.30 & 3.568 & 18.29 & 5019 \\
\hline 500 & 0.42 & 1.95 & 4.00 & 42.01 & 43.29 & 53.13 & 3.689 & 18.65 & 5217 \\
\hline
\end{tabular}

6.4. Optimal Solution Changing with Attenuation Coefficient $\alpha$. We have shown that when $\alpha \rightarrow \infty$, the optimal solution of our model converges to the optima of the basic EOQPBO model (Corollary 7 ). Let $T_{\infty}, F_{\infty}$, and $\Gamma_{\infty}$ represent the optimal order cycle, the optimal fill rate, and the corresponding optimal inventory cost when $\alpha \rightarrow \infty$, which can be obtained by solving the basic EOQ-PBO model [10]. Correspondingly, let $T_{\alpha}, F_{\alpha}$, and $\Gamma_{\alpha}$ indicate the optimal order cycle, the optimal fill rate, and the optimal inventory cost for a limited value of $\alpha<\infty$. Densify $\alpha$ by, respectively, setting $\alpha=0.1,0.25,0.5,1,3,5,10,25,50,75,100,300,500$. Implementing the scheme in Section 6.2 to generate numerical examples, each value of $\alpha$ corresponds to $A \times C_{h} \times C_{b} \times$ $C_{o} \times \beta \times D=4 \times 4 \times 4 \times 4 \times 5 \times 4=5120$ problem instances. Solving all the numerical examples by Opt_Global for each $\alpha$, the average, minimum, and maximum values of the ratio of $F_{\alpha} / F_{\infty}$ and $T_{\alpha} / T_{\infty}$ are presented in Figure 5.

It can be observed that $F_{\alpha}$ is decreasing in $\alpha$ until reaching $F_{\infty}$ when $\alpha \rightarrow \infty\left(F_{\alpha} / F_{\infty} \rightarrow 1\right)$, which has an intuitive interpretation: A smaller $\alpha$ corresponds to a smaller backorder demand rate so that the unsatisfied customer of the previous order cycle will fetch the backordered item later. In this case, the store must keep the backordered item for a longer time and incur a larger inventory holding cost. Thus, if $\alpha$ is smaller, the vendor should backorder less resulting in a larger fill rate, to avoid too much inventory holding cost of the backordered demand. Conversely, if $\alpha$ increases, the optimal fill rate $F_{\alpha}$ decreases.

Moreover, another method for avoiding a high inventory holding cost caused by the backordered demand in case of

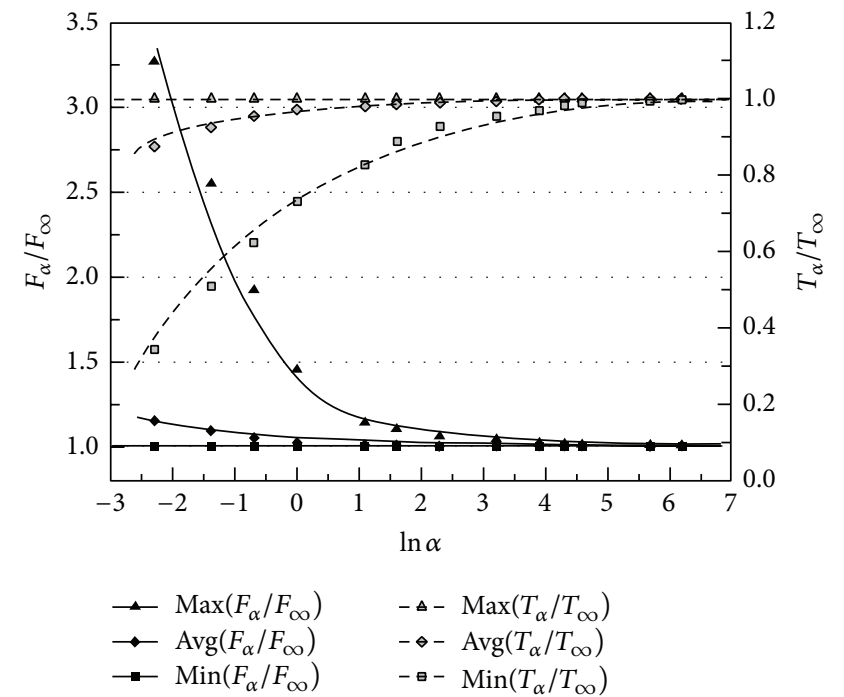

Figure 5: The ratio of the optimal solution, $F_{\alpha} / F_{\infty}$ and $T_{\alpha} / T_{\infty}$, under different $\alpha$.

a small $\alpha$ is to diminish the maximum backorder level by promising a shorter stockout period. Intuitively, this can be realized by replenishing inventory more frequently based on a shorter order cycle. It follows that, under a smaller $\alpha$, a shorter order cycle is more preferable. In other words, $T_{\alpha}$ is increasing in $\alpha$, which can also be observed in Figure 5. 


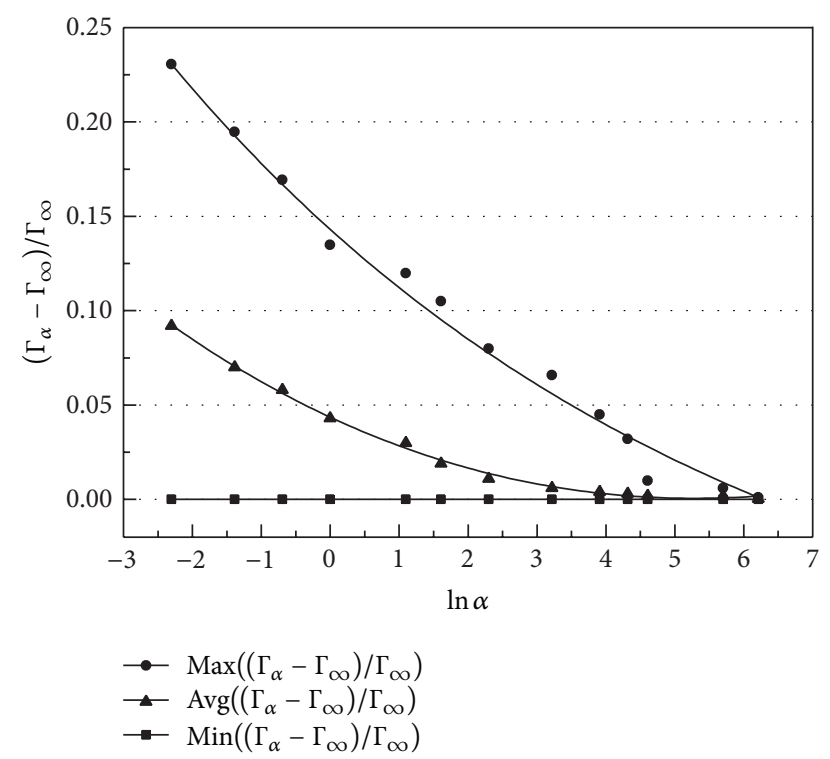

FIGURE 6: The optimal inventory cost decreases in $\alpha$ until reaching that of the basic EOQ-PBO.

In addition, Figure 5 shows that, for some numerical examples, the optimal order cycle and the fill rate are always equal to their counterparts of the basic EOQ-PBO $\left(T_{\alpha} / T_{\infty}\right.$ and $F_{\alpha} / F_{\infty}$ are equal to 1$)$. We have checked these examples and found that if the basic EOB-PBO's optimal policy is $F_{\infty}=$ 1 , that is, to meet all demands without any lost sales, then $F_{\alpha}$ is also equal to 1 , no matter what value of $\alpha$ is. In fact, since $F_{\alpha}$ is decreasing in $\alpha$ and $F_{\infty} \leq F_{\alpha} \leq 1$, it is an inevitable outcome that $F_{\alpha}$ must be equal to 1 in case of $F_{\infty}=1$.

Figure 6 presents the relative deviations of the optimal inventory cost $\Gamma_{\alpha}$ from $\Gamma_{\infty}$, where $\Gamma_{\infty}$ represents the optimal inventory cost of the basic EOQ-PBO model.

We can see that the inventory cost is decreasing in $\alpha$, and the optimal solution converges quickly to the case of $\alpha \rightarrow \infty$ as $\alpha$ increases. In particular, when $\ln \alpha>3.4$, that is, $\alpha>30$, the optimal solution will be sufficiently close to that of the basic EOQ-PBO with a relative deviation less than $5 \%$. That means if the backordered customers revisit the store without too much purchase delay, the basic EOQ-PBO can approximate our model very well.

\section{Conclusions}

It is frequent that if demands are not filled and the unsatisfied customers are willing to accept the backorder, she/he will fetch the demanded item in the next order cycle. The basic EOQ with partial backordering assumes that all backordered customers will fetch their products simultaneously at the beginning of the very next order cycle and the store will not incur inventory holding cost for the backordered demand. However, this assumption may be violated in some cases where the backordered customer may revisit the store successively because of the purchase delay, and therefore the backordered demand is not filled instantly.
This paper proposed a new inventory model with partial backordering by assuming that the backordered customers repurchase the backordered products successively and the arriving rate of the backordered customer is proportional to the backordered customers who have not been filled. This leads to an exponentially decreasing demand rate in time regarding the backordered demand, based on which we formulated the total inventory cost function. To solve the model, we developed an efficient algorithm based on Lipschitz Optimization and conduct numerical experiments proving the efficiency and effectiveness of the algorithm.

From the theoretical results and numerical computations, we mainly find that (1) the larger the attenuation coefficient of the backorder demand rate, the lower the fill rate with which the store will satisfy the customer to avoid holding backordered items for a longer time resulting in higher inventory holding cost; (2) the more slowly the backordered customer revisits the store, the shorter the order cycle that the store should set is, to diminish the backorder level and therefore decrease the inventory holding cost of the backordered demand; (3) a larger backorder demand attenuation coefficient leads to a smaller total inventory cost, so the store should deliver the item to the backordered customers as soon as possible.

Compared with the traditional EOQ-PBO model, the new parameter needed when implementing our model in the real-world is the attenuation coefficient $\alpha . \alpha$ reflects the proportion of the backordered customer who has revisited the store and fetched the backordered item. To estimate this parameter, the vendor need record the number of the backordered customers who revisit the store per unit time, which is compared with the total number of the backordered customers of the previous stockout. Thereby, the attenuation coefficient $\alpha$ can be calibrated.

The study can be extended by combining the limited backorder demand rate considered in this paper with other inventory problems, for example, deteriorating items, timevarying demand rate, and the vendor-retailer supply chain, to further enrich the study of EOQ-PBO models. The consumer behavior model describing how the backordered customers repurchase their demanded items also deserves more researches. In addition, this paper mainly concentrates on the theoretical analysis of the new inventory problem. To implement a theoretical inventory model based on practical data is very important for the research on operations management, which will be carried out as the empirical study in the future work.

\section{Appendices}

\section{A. Lemmas}

For the convenience of proof, we first present some properties of function $\theta(x)=x\left(e^{x}-1\right)^{-1}$, which are straightforward by L'Hôpital's rule (Bernoulli's rule), as Lemmas A.1 and A.2.

Lemma A.1. $\theta(x)$ is decreasing and convex in $x \in(0, \infty)$, and $\lim _{x \rightarrow 0} \theta(x)=1, \lim _{x \rightarrow \infty} \theta(x)=0$. 

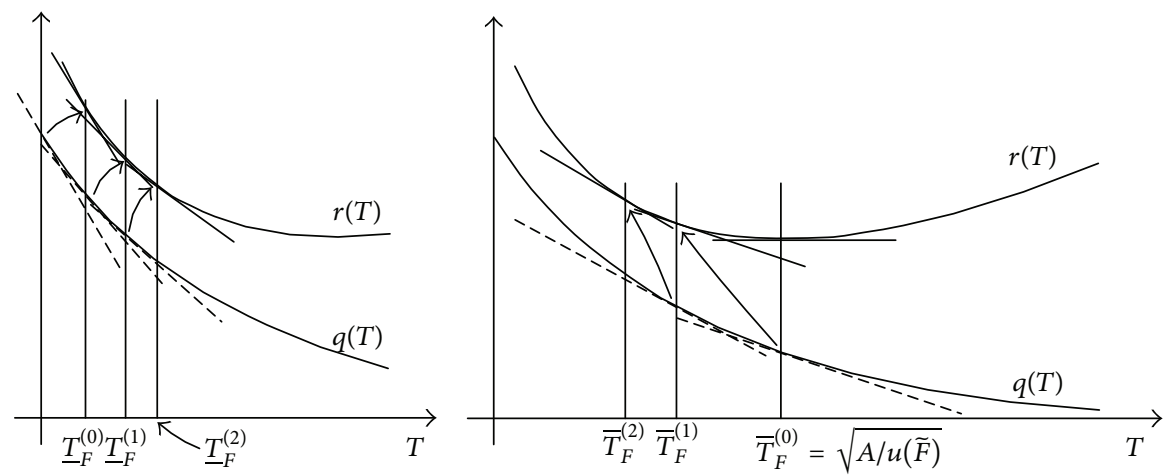

FIGURE 7: The curves of functions $r(T)$ and $q(T)$.

Lemma A.2. For $x \in(0, \infty)$, regarding the derivatives of $\theta(x)$ with respect to $x$, one has

$$
\begin{aligned}
& \lim _{x \rightarrow 0} \theta^{\prime}(x)=-\frac{1}{2}, \\
& \lim _{x \rightarrow \infty} \theta^{\prime}(x)=0, \\
& \theta^{\prime}(x) \leq 0 ; \\
& \lim _{x \rightarrow 0} \theta^{\prime \prime}(x)=\frac{1}{6}, \\
& \lim _{x \rightarrow \infty} \theta^{\prime \prime}(x)=0, \\
& \theta^{\prime \prime}(x) \geq 0 .
\end{aligned}
$$

Moreover, regarding function $q(T)=v(\widetilde{F}) \theta(\alpha \widetilde{F} T)$, Lemma A.3 can be easily proved based on Lemma A.2, as shown below.

Lemma A.3. $q(T)$ is decreasing and convex in $T$ with given F. In particular, $\lim _{T \rightarrow 0} q(T)=v(\widetilde{F})$ and $\lim _{T \rightarrow \infty} q(T)=0$; $\lim _{T \rightarrow 0} q^{\prime}(T)=-\alpha \widetilde{F} \cdot v(\widetilde{F}) / 2$ and $\lim _{T \rightarrow \infty} q^{\prime}(T)=0$.

\section{B. Proofs}

Proof of Proposition 4. From Lemma A.3, the curves of function $r(T)$ and function $q(T)$ are as in Figure 7.

The right side of Figure 7 shows that the optimal value of $T$ should not be larger than the optimum of function $r(T)=$ $A / T+u(\widetilde{F}) T$; that is,

$$
\widehat{T}_{F} \leq \bar{T}_{F}^{(0)}=\sqrt{\frac{A}{u(\widetilde{F})}} .
$$

Note that $q(T)$ is decreasing and convex in $T$. Thus, if $\widehat{T}_{F} \leq \bar{T}_{F}^{(0)}$, then on the optimal point $T=\widehat{T}_{F}$, the absolute value of the first-order derivative of $q(T)$ must not be smaller than the first-order derivative on $T=\bar{T}_{F}^{(0)}$; that is, $\left|q^{\prime}\left(\widehat{T}_{F}\right)\right| \geq$ $\left|q^{\prime}\left(\bar{T}_{F}^{(0)}\right)\right|$. Proposition 3 shows that on the optimal point we have $r^{\prime}\left(\widehat{T}_{F}\right)=q^{\prime}\left(\widehat{T}_{F}\right)$. Thus, $\left|r^{\prime}\left(\widehat{T}_{F}\right)\right|=\left|q^{\prime}\left(\widehat{T}_{F}\right)\right| \geq\left|q^{\prime}\left(\bar{T}_{F}^{(0)}\right)\right|$; that is,

$$
\left|-\frac{A}{\widehat{T}_{F}^{2}}+u(\widetilde{F})\right| \geq\left|\alpha \widetilde{F} \cdot v(\widetilde{F}) \cdot \theta^{\prime}\left(\bar{x}^{(0)}\right)\right|,
$$

where $\bar{x}^{(0)}=\alpha \widetilde{F} \bar{T}_{F}^{(0)}$. Because $r^{\prime}(T) \leq 0$, that is, $-A / \widehat{T}_{F}^{2}+$ $u(\widetilde{F}) \leq 0$ if $T \leq \bar{T}_{F}^{(0)}$, and $\theta^{\prime}(x)<0$, it follows that

$$
\frac{A}{\widehat{T}_{F}^{2}}-u(\widetilde{F}) \geq-\alpha \widetilde{F} \cdot v(\widetilde{F}) \cdot \theta^{\prime}\left(\bar{x}^{(0)}\right) .
$$

Hence, we have

$$
\widehat{T}_{F} \leq \sqrt{\frac{A}{u(\widetilde{F})-\alpha \widetilde{F} \cdot v(\widetilde{F}) \cdot \theta^{\prime}\left(\bar{x}^{(0)}\right)}} .
$$

Denote

$$
\bar{T}_{F}^{(1)}=\sqrt{\frac{A}{u(\widetilde{F})-\alpha \widetilde{F} \cdot v(\widetilde{F}) \cdot \theta^{\prime}\left(\bar{x}^{(0)}\right)}} .
$$

Note that $\theta^{\prime}(x)<0$, so $\bar{T}_{F}^{(1)}<\bar{T}_{F}^{(0)}$ and the upper bound of $\widehat{T}_{F}$ is diminished.

Similarly, the absolute value of the first derivative of $q(T)$ on the optimal point $T=\widehat{T}_{F}$ must not be smaller than the derivative on $T=\bar{T}_{F}^{(1)}$; that is, $\left|q^{\prime}\left(\widehat{T}_{F}\right)\right| \geq\left|q^{\prime}\left(\bar{T}_{F}^{(1)}\right)\right|$. By Proposition 3 that $r^{\prime}\left(\widehat{T}_{F}\right)=q^{\prime}\left(\widehat{T}_{F}\right)$, we have $\left|r^{\prime}\left(\widehat{T}_{F}\right)\right|=$ $\left|q^{\prime}\left(\widehat{T}_{F}\right)\right| \geq\left|q^{\prime}\left(\bar{T}_{F}^{(1)}\right)\right|$, which yields

$$
\widehat{T}_{F} \leq \bar{T}_{F}^{(2)}=\sqrt{\frac{A}{u(\widetilde{F})-\alpha \widetilde{F} \cdot v(\widetilde{F}) \cdot \theta^{\prime}\left(\bar{x}^{(1)}\right)}},
$$

where $\bar{x}^{(1)}=\alpha \widetilde{F} \bar{T}_{F}^{(1)}$.

From Lemma A.2, $\theta^{\prime}(x)$ is strictly increasing in $x<\infty$ because $\theta^{\prime \prime}(x)>0$ for a limited value of $x$. As mentioned above, $\bar{T}_{F}^{(1)}<\bar{T}_{F}^{(0)}$ implying that $\bar{x}^{(1)}<\bar{x}^{(0)}$, so $\theta^{\prime}\left(\bar{x}^{(1)}\right)<$ $\theta^{\prime}\left(\bar{x}^{(0)}\right)$. As a result, we have $u(\widetilde{F})-\alpha \widetilde{F} \cdot v(\widetilde{F}) \cdot \theta^{\prime}\left(\bar{x}^{(1)}\right)>$ $u(\widetilde{F})-\alpha \widetilde{F} \cdot v(\widetilde{F}) \cdot \theta^{\prime}\left(\bar{x}^{(0)}\right)$ and it follows that $\bar{T}_{F}^{(2)}<\bar{T}_{F}^{(1)}$. 
The upper bound of $\widehat{T}_{F}$ is again diminished. Repeating the above derivation we can obtain a decreasing sequence $\left\{\bar{T}_{F}^{(k)}\right\}$ that bounds the maximum value of $\widehat{T}_{F}$, as follows:

$$
\widehat{T}_{F} \leq \bar{T}_{F}^{(k)}=\sqrt{\frac{A}{u(\widetilde{F})-\alpha \widetilde{F} \cdot v(\widetilde{F}) \cdot \theta^{\prime}\left(\bar{x}^{(k-1)}\right)}},
$$

where $\bar{x}^{(k-1)}=\alpha \widetilde{F} \bar{T}_{F}^{(k-1)},(k=1, \ldots, \infty)$, and $\bar{T}_{F}^{(0)}=$ $\sqrt{A / u(\widetilde{F})}$

Obviously, $\bar{T}_{F}^{(k)}$ of any $k$ gives an upper bound for $\widehat{T}_{F}$, and $\bar{T}_{F}=\lim _{k \rightarrow \infty} \bar{T}_{F}^{(k)}$ is the best upper bound of $\widehat{T}_{F}$. The proof is completed.

Proof of Proposition 5. Because function $q(T)$ is convex and decreasing in $T$, from the left side of Figure 7 we can see that the absolute value of $q^{\prime}(T)$ reaches its maximum value if $T \rightarrow 0$. Thus, $\left|q^{\prime}(T)\right| \leq\left|\lim _{T \rightarrow 0} q^{\prime}(T)\right|$. Proposition 3 shows that on the optimal point $T=\widehat{T}_{F}$ we have $r^{\prime}\left(\widehat{T}_{F}\right)=q^{\prime}\left(\widehat{T}_{F}\right)$. Thus, $\left|r^{\prime}\left(\widehat{T}_{F}\right)\right|=\left|q^{\prime}\left(\widehat{T}_{F}\right)\right| \leq\left|\lim _{T \rightarrow 0} q^{\prime}(T)\right|$. In addition, from Lemma A.3 we have $\lim _{T \rightarrow 0} q^{\prime}(T)=-\alpha \widetilde{F} \cdot v(\widetilde{F}) / 2$ so that

$$
\left|-\frac{A}{\widehat{T}_{F}^{2}}+u(\widetilde{F})\right| \leq\left|-\alpha \widetilde{F} \cdot \frac{v(\widetilde{F})}{2}\right| .
$$

Note that $-A / \widehat{T}_{F}^{2}+u(\widetilde{F})<0$, which yields

$$
\widehat{T}_{F} \geq \underline{T}_{F}^{(0)}=\sqrt{\frac{2 A}{2 u(\widetilde{F})+\alpha \widetilde{F} \cdot v(\widetilde{F})}} .
$$

Thus, $\underline{T}_{F}^{(0)}$ is a lower bound of $\widehat{T}_{F}$. Furthermore, because $q(T)$ is decreasing and convex in $T$, then, on the optimal point $T=\widehat{T}_{F}$, the absolute value of the first derivative of $q(T)$ must not be larger than that on $T=\underline{T}_{F}^{(0)}$; that is, $\left|q^{\prime}\left(\widehat{T}_{F}\right)\right| \leq$ $\left|q^{\prime}\left(\underline{T}_{F}^{(0)}\right)\right|$. By Proposition 3 that $r^{\prime}\left(\widehat{T}_{F}\right)=q^{\prime}\left(\widehat{T}_{F}\right)$ we have $\left|r^{\prime}\left(\widehat{T}_{F}\right)\right|=\left|q^{\prime}\left(\widehat{T}_{F}\right)\right| \leq\left|q^{\prime}\left(\underline{T}_{F}^{(0)}\right)\right|$; that is,

$$
\left|-\frac{A}{\widehat{T}_{F}^{2}}+u(\widetilde{F})\right| \leq\left|\alpha \widetilde{F} \cdot v(\widetilde{F}) \theta^{\prime}\left(\underline{x}^{(0)}\right)\right|,
$$

where $\underline{x}^{(0)}=\alpha \widetilde{F} \underline{T}_{F}^{(0)}$. The above inequality yields

$$
\frac{A}{\widehat{T}_{F}^{2}}-u(\widetilde{F}) \leq-\alpha \widetilde{F} \cdot v(\widetilde{F}) \cdot \theta^{\prime}\left(\underline{x}^{(0)}\right) .
$$

Hence, we have

$$
\widehat{T}_{F} \geq \underline{T}_{F}^{(1)}=\sqrt{\frac{A}{u(\widetilde{F})-\alpha \widetilde{F} \cdot v(\widetilde{F}) \cdot \theta^{\prime}\left(\underline{x}^{(0)}\right)}}
$$

Lemma A. 2 shows that $-1 / 2<\theta^{\prime}(x)<0$. It follows that $u(\widetilde{F})-\alpha \widetilde{F} \cdot v(\widetilde{F}) \cdot \theta^{\prime}\left(\underline{x}^{(0)}\right)<u(\widetilde{F})+\alpha \widetilde{F} \cdot v(\widetilde{F}) / 2$, so we have $\underline{T}_{F}^{(1)}>$ $\underline{T}_{F}^{(0)}$. The lower bound of $\widehat{T}_{F}$ is increased by $\underline{T}_{F}^{(1)}$. Repeating the above procedure, (18) yields an increasing sequence $\left\{\underline{T}_{F}^{(k)}\right\}$ that bounds the minimum value of $\widehat{T}_{F}$ as

$$
\widehat{T}_{F} \geq \underline{T}_{F}^{(k)}=\sqrt{\frac{A}{u(\widetilde{F})-\alpha \widetilde{F} \cdot v(\widetilde{F}) \cdot \theta^{\prime}\left(\underline{x}^{(k-1)}\right)}},
$$

where $\underline{x}^{(k-1)}=\alpha \widetilde{F} \underline{T}_{F}^{(k-1)},(k=1, \ldots, \infty)$, and $\underline{T}_{F}^{(0)}=$ $\sqrt{2 A /(2 u(\widetilde{F})+\alpha \widetilde{F} \cdot v(\widetilde{F}))} \cdot \underline{T}_{F}=\underline{T}_{F}^{(k)}$ of any $k$ can be a lower bound of $\widehat{T}_{F}$ and $\underline{T}_{F}=\lim _{k \rightarrow \infty} \underline{T}_{F}^{(k)}$ is the best lower bound. The proof is completed.

Proof of Proposition 6. For the sequence of the upper bound $\bar{T}_{F}^{(k)}=\sqrt{A /\left(u(\widetilde{F})-\alpha \widetilde{F} \cdot v(\widetilde{F}) \cdot \theta^{\prime}\left(\bar{x}^{(k-1)}\right)\right)}$, if the limit exists, we have

$$
\begin{aligned}
\bar{T}_{F} & =\sqrt{\frac{A}{u(\widetilde{F})-\alpha \widetilde{F} \cdot v(\widetilde{F}) \cdot \theta^{\prime}(\bar{x})}} \\
& =\sqrt{\frac{A}{u(\widetilde{F})-\alpha \widetilde{F} \cdot v(\widetilde{F}) \cdot \theta^{\prime}\left(\alpha \widetilde{F} \bar{T}_{F}\right)}},
\end{aligned}
$$

which is recast as

$$
\begin{gathered}
-\frac{A}{\bar{T}_{F}^{2}}+u(\widetilde{F})-\alpha \widetilde{F} \cdot v(\widetilde{F}) \cdot \theta^{\prime}\left(\alpha \widetilde{F} \bar{T}_{F}\right) \\
=r^{\prime}\left(\bar{T}_{F}\right)-q^{\prime}\left(\bar{T}_{F}\right)=0 .
\end{gathered}
$$

Thus, the limit of the upper bound solves the first-order necessary condition. Similarly, it can be proved the limit of the lower bound also solves the first-order necessary condition. This completes the proof.

Proof of Proposition 9. Suppose that function $f(x)$ and its first-order derivative $f^{\prime}(x)$ are continuous on interval $[a, b]$; that is, $f(x), f^{\prime}(x) \in \mathbf{C}[a, b]$. According to Theorem 2.2 in Mathews and Fink [50], if $\left|f^{\prime}(x)\right| \leq \mathscr{L}<1$ ( $\mathscr{L}$ is a real number) and $f(x) \in[a, b]$ for all $x \in[a, b]$, then the fixedpoint iteration $p_{n}=f\left(p_{n-1}\right)$ converges to the unique fixed point $P \in[a, b]$.

From Propositions 4 and 5, the iterative formula of the upper and lower bounds is

$$
T_{F}^{(k)}=\sqrt{\frac{A}{u(\widetilde{F})-\alpha \widetilde{F} \cdot v(\widetilde{F}) \cdot \theta^{\prime}\left(\alpha \widetilde{F} T_{F}^{(k-1)}\right)}},
$$

$$
(k=1, \ldots, \infty) \text {. }
$$

Define a function as $\phi(T)=\sqrt{A /\left(u(\widetilde{F})-\alpha \widetilde{F} \cdot v(\widetilde{F}) \cdot \theta^{\prime}(\alpha \widetilde{F} T)\right)}$. Obviously, the limit of $T_{F}^{(k)}$ is the fixed point of function $\phi(T)$. Note that, from Propositions 4 and 5, we have $T \in\left[\underline{T}_{F}, \bar{T}_{F}\right]$. Propositions 4 and 5 show that $\underline{T}_{F}^{(k+1)} \geq \underline{T}_{F}^{(k)}$ and $\bar{T}_{F}^{(k+1)} \leq \bar{T}_{F}^{(k)}$, so $\underline{T}_{F} \leq \phi(T) \leq \bar{T}_{F}$ for all $T \in\left[\underline{T}_{F}, \bar{T}_{F}\right]$. 
Moreover, $\phi^{\prime}(T)=\left(\alpha^{2} \widetilde{F}^{2} v(\widetilde{F}) \sqrt{A} / 2\right)[u(\widetilde{F}) \quad-$ $\left.\alpha \widetilde{F} v(\widetilde{F}) \theta^{\prime}(\alpha \widetilde{F} T)\right]^{-3 / 2} \theta^{\prime \prime}(\alpha \widetilde{F} T)$. Lemma A.2 shows that $\theta^{\prime}(\alpha \widetilde{F} T) \leq 0$ and $0 \leq \theta^{\prime \prime}(\alpha \widetilde{F} T) \leq 1 / 6$, so

$$
\begin{aligned}
0 & \leq \phi^{\prime}(T) \\
& \leq \frac{\alpha^{2} \widetilde{F}^{2} v(\widetilde{F}) \sqrt{A}}{12}\left[u(\widetilde{F})-\alpha \widetilde{F} v(\widetilde{F}) \theta^{\prime}(\alpha \widetilde{F} T)\right]^{-3 / 2} .
\end{aligned}
$$

According to Lemma A.2, $\theta^{\prime}(\alpha \widetilde{F} T)$ is increasing in $T$, so

$$
\begin{gathered}
\phi^{\prime}(T) \leq \frac{\alpha^{2} \widetilde{F}^{2} v(\widetilde{F}) \sqrt{A}}{12}[u(\widetilde{F}) \\
\left.-\alpha \widetilde{F} v(\widetilde{F}) \theta^{\prime}(\alpha \widetilde{F} T)\right]^{-3 / 2} \\
\leq \frac{\alpha^{2} \widetilde{F}^{2} v(\widetilde{F}) \sqrt{A}}{12}[u(\widetilde{F}) \\
\left.-\alpha \widetilde{F} v(\widetilde{F}) \theta^{\prime}\left(\alpha \widetilde{F} \bar{T}_{F}\right)\right]^{-3 / 2} .
\end{gathered}
$$

As mentioned above, for function $\phi(T)$, if $\left|\phi^{\prime}(T)\right| \leq \mathscr{L}<$ 1 , then its fixed point is unique. Note that $\phi^{\prime}(T) \geq 0$, so a sufficient condition ensuring $\phi^{\prime}(T) \leq \mathscr{L}<1$ is

$$
\begin{aligned}
& \frac{\alpha^{2} \widetilde{F}^{2} v(\widetilde{F}) \sqrt{A}}{12}\left[u(\widetilde{F})-\alpha \widetilde{F} v(\widetilde{F}) \theta^{\prime}\left(\alpha \widetilde{F} \bar{T}_{F}\right)\right]^{-3 / 2} \\
& <1,
\end{aligned}
$$

which yields $\alpha^{2} \widetilde{F}^{2} v(\widetilde{F}) \sqrt{A}<12\left[u(\widetilde{F})-\alpha \widetilde{F} v(\widetilde{F}) \theta^{\prime}\left(\alpha \widetilde{F} \bar{T}_{F}\right)\right]^{3 / 2}$. This is (19). The proof is completed.

Proof of Proposition 10. With given $F$, the first-order derivative of the inventory cost function with respect to $T$ is

$$
\begin{aligned}
\frac{d \widetilde{\Gamma}(T)}{d T} & =r^{\prime}(T)-q^{\prime}(T) \\
& =-\frac{A}{T^{2}}+u(\widetilde{F})-\alpha \widetilde{F} \cdot v(\widetilde{F}) \cdot \theta^{\prime}(\alpha \widetilde{F} T) .
\end{aligned}
$$

Considering that $-1 / 2 \leq \theta^{\prime}(x) \leq 0$, if $d \widetilde{\Gamma}(T) / d T \geq 0$, the maximum value of $d \widetilde{\Gamma}(T) / d T$ is $u(\widetilde{F})+\alpha \widetilde{F} \cdot v(\widetilde{F}) / 2-A / \bar{T}_{F}^{2}$. If $d \widetilde{\Gamma}(T) / d T \leq 0$, the minimum value of $d \widetilde{\Gamma}(T) / d T$ is $u(\widetilde{F})-$ $A / \underline{T}_{F}^{2}$, so that the absolute value of $d \widetilde{\Gamma}(T) / d T$ meets

$$
\begin{aligned}
\left|\frac{d \widetilde{\Gamma}(T)}{d T}\right| & \leq\left|u(\widetilde{F})+\frac{\alpha \widetilde{F} \cdot v(\widetilde{F})}{2}-\frac{A}{\bar{T}_{F}^{2}}\right| \\
& \text { or }\left|\frac{d \widetilde{\Gamma}(T)}{d T}\right| \leq\left|u(\widetilde{F})-\frac{A}{T_{F}^{2}}\right| .
\end{aligned}
$$

Hence, the Lipschitz constant for the model with given $F$ can be taken as

$$
\begin{gathered}
L_{\widetilde{F}}=\max \left\{\left|u(\widetilde{F})+\frac{\alpha \widetilde{F} \cdot v(\widetilde{F})}{2}-\frac{A}{\bar{T}_{F}^{2}}\right|,\right. \\
\left.\left|u(\widetilde{F})-\frac{A}{T_{F}^{2}}\right|\right\} .
\end{gathered}
$$

This completes the proof.

\section{Notations}

$D: \quad$ The routine demand rate, that is, the demand rate of the first time visiting customer who has not incurred any stockouts, in units/unit time

A: $\quad$ The ordering cost for placing and receiving an order, in \$/order

$C_{o}$ : The opportunity cost of unit lost sale, including the lost profit and goodwill loss, in \$/unit

$C_{h}$ : The cost to hold one unit item for one unit time, in \$/unit/unit time

$C_{b}$ : The cost to keep one unit backordered for one unit time, in \$/unit/unit time

$\alpha$ : Attenuation coefficient of the backorder demand rate

$\beta$ : $\quad$ The backordering rate, that is, the fraction of stockouts that will be backordered

T: $\quad$ The order cycle, that is, the time interval between two replenishments

$F$ : The fill rate, that is, the percentage of the routine demand that is filled from the shelf stock

$\bar{B}$ : $\quad$ The maximum backorder level during one stockout period, in units

$t$ : The time elapses from the beginning of an order cycle

$\mathscr{B}(t)$ : The unsatisfied backordered demand remained at time $t$ during an in-stock period, in units

$I_{B}(t)$ : The inventory level corresponding to $\mathscr{B}(t)$, in units

$D_{b}(t):$ The backorder demand rate at time $t$, which is generated by the backordered customers who have not been satisfied, in units/unit time.

\section{Conflict of Interests}

The authors declare that there is no conflict of interests regarding the publication of this paper.

\section{Acknowledgments}

The research of Ren-Qian Zhang is supported by NSFC under 71271010 and 71571006, and the research of Wen-Hui Zhou is supported by NSFC under 71271089. 


\section{References}

[1] D. C. Montgomery, M. S. Bazaraa, and A. K. Keswani, "Inventory models with a mixture of backorders and lost sales," Naval Research Logistics Quarterly, vol. 20, no. 2, pp. 255-263, 1973.

[2] D. W. Pentico and M. J. Drake, "A survey of deterministic models for the EOQ and EPQ with partial backordering," European Journal of Operational Research, vol. 214, no. 2, pp. 179-198, 2011.

[3] W. Zinn and P. C. Liu, "Consumer response to retail stockouts," Journal of Business Logistics, vol. 22, no. 1, pp. 49-71, 2001.

[4] G. Liberopoulos and I. Tsikis, "Do stockouts undermine immediate and future sales? Statistical evidence from a wholesaler's historical data," Working Paper, Department of Mechanical Engineering, University of Thessaly, Volos, Greece, 2008.

[5] S. Rao, S. E. Griffis, and T. J. Goldsby, "Failure to deliver? Linking online order fulfillment glitches with future purchase behavior," Journal of Operations Management, vol. 29, no. 7-8, pp. 692-703, 2011.

[6] D. Rosenberg, "A new analysis of a lot-size model with partial backordering," Naval Research Logistics Quarterly, vol. 26, no. 2, pp. 349-353, 1979.

[7] K. S. Park, "Inventory model with partial backorders," International Journal of Systems Science, vol. 13, no. 12, pp. 1313-1317, 1982.

[8] K. S. Park, "Another inventory model with a mixture of backorders and lost sales," Naval Research Logistics Quarterly, vol. 30, no. 3, pp. 397-400, 1983.

[9] H. M. M. Wee, "Optimal inventory policy with partial backordering," Optimal Control Applications and Methods, vol. 10, no. 2, pp. 181-187, 1989.

[10] D. W. Pentico and M. J. Drake, "The deterministic EOQ with partial backordering: a new approach," European Journal of Operational Research, vol. 194, no. 1, pp. 102-113, 2009.

[11] N. E. Abboud and R. G. Sfairy, "Time-limited free back-orders EOQ model," Applied Mathematical Modelling, vol. 21, no. 1, pp. 21-25, 1997.

[12] L. A. San José, J. Sicilia, and J. García-Laguna, "The lot sizereorder level inventory system with customers impatience functions," Computers \& Industrial Engineering, vol. 49, no. 3, pp. 349-362, 2005.

[13] L. A. San José, J. Sicilia, and J. García-Laguna, "Analysis of an inventory system with exponential partial backordering," International Journal of Production Economics, vol. 100, no. 1, pp. 76-86, 2006.

[14] L. A. San-José, J. Sicilia, and J. García-Laguna, "An economic lot-size model with partial backlogging hinging on waiting time and shortage period," Applied Mathematical Modelling, vol. 31, no. 10, pp. 2149-2159, 2007.

[15] C.-Y. Dye and T.-P. Hsieh, "An optimal replenishment policy for deteriorating items with effective investment in preservation technology," European Journal of Operational Research, vol. 218, no. 1, pp. 106-112, 2012.

[16] H.-M. Wee, "A deterministic lot-size inventory model for deteriorating items with shortages and a declining market," Computers \& Operations Research, vol. 22, no. 3, pp. 345-356, 1995.

[17] K. Skouri and S. Papachristos, "A continuous review inventory model, with deteriorating items, time-varying demand, linear replenishment cost, partially time-varying backlogging," Applied Mathematical Modelling, vol. 26, no. 5, pp. 603-617, 2002.
[18] Y.-W. Zhou, H.-S. Lau, and S.-L. Yang, "A finite horizon lot-sizing problem with time-varying deterministic demand and waiting-time-dependent partial backlogging," International Journal of Production Economics, vol. 91, no. 2, pp. 109-119, 2004.

[19] S. K. Ghosh and K. S. Chaudhuri, "An EOQ model for a deteriorating item with trended demand and variable backlogging with shortages in all cycles," Advanced Modeling and Optimization, vol. 7, no. 1, pp. 57-68, 2005.

[20] N. H. Shah, "Store's optimal policy for time-dependent deteriorating inventory with exponentially decreasing demand and partial backlogging," ASOR Bulletin, vol. 28, no. 2, pp. 9-14, 2009.

[21] J.-T. Teng, J. Min, and Q. Pan, "Economic order quantity model with trade credit financing for non-decreasing demand," Omega, vol. 40, no. 3, pp. 328-335, 2012.

[22] B. Sarkar, "An EOQ model with delay in payments and time varying deterioration rate," Mathematical and Computer Modelling, vol. 55, no. 3-4, pp. 367-377, 2012.

[23] G. Padmanabhan and P. Vrat, "EOQ models for perishable items under stock dependent selling rate," European Journal of Operational Research, vol. 86, no. 2, pp. 281-292, 1995.

[24] K.-L. Hou, "An inventory model for deteriorating items with stock-dependent consumption rate and shortages under inflation and time discounting," European Journal of Operational Research, vol. 168, no. 2, pp. 463-474, 2006.

[25] S. Jain, M. Kumar, and P. Advani, "An inventory model with inventory level dependent demand rate, deterioration, partial backlogging and decrease in demand," International Journal of Operations Research, vol. 5, no. 3, pp. 154-159, 2008.

[26] R. Uthayakumar and K. V. Geetha, "A replenishment policy for non-instantaneous deteriorating inventory system with partial backlogging," Tamsui Oxford Journal of Mathematical Sciences, vol. 25, no. 3, pp. 313-332, 2009.

[27] H.-L. Yang, J.-T. Teng, and M.-S. Chern, "An inventory model under inflation for deteriorating items with stock-dependent consumption rate and partial backlogging shortages," International Journal of Production Economics, vol. 123, no. 1, pp. 8-19, 2010.

[28] P. L. Abad, "Optimal pricing and lot-sizing under conditions of perishability and partial backordering," Management Science, vol. 42, no. 8, pp. 1093-1104, 1996.

[29] P. L. Abad, "Optimal pricing and lot-sizing under conditions of perishability, finite production and partial backordering and lost sale," European Journal of Operational Research, vol. 144, no. 3, pp. 677-685, 2003.

[30] A. A. Taleizadeh, D. W. Pentico, M. S. Jabalameli, and M. Aryanezhad, "An economic order quantity model with multiple partial prepayments and partial backordering," Mathematical and Computer Modelling, vol. 57, no. 3-4, pp. 311-323, 2013.

[31] A. A. Taleizadeh, D. W. Pentico, M. Saeed Jabalameli, and M. Aryanezhad, "An EOQ model with partial delayed payment and partial backordering," Omega, vol. 41, no. 2, pp. 354-368, 2013.

[32] A. A. Taleizadeh and D. W. Pentico, "An Economic Order Quantity model with partial backordering and all-units discount," International Journal of Production Economics, vol. 155, pp. 172184, 2014.

[33] H.-M. Wee, Y.-D. Huang, W.-T. Wang, and Y.-L. Cheng, "An EPQ model with partial backorders considering two backordering costs," Applied Mathematics and Computation, vol. 232, pp. 898-907, 2014. 
[34] S. Duran, T. Liu, D. Simchi-Levi, and J. L. Swann, "Policies utilizing tactical inventory for service-differentiated customers," Operations Research Letters, vol. 36, no. 2, pp. 259-264, 2008.

[35] E. A. Greenleaf and D. R. Lehmann, "Reasons for substantial delay in consumer decision making," The Journal of Consumer Research, vol. 22, no. 2, pp. 186-199, 1995.

[36] G.-R. Chen and M.-L. Chen, "The link between purchase delay and resale price maintenance: using the real options approach," International Journal of Economics and Finance, vol. 3, no. 1, pp. 171-177, 2011.

[37] I. Ajzen, "From intentions to actions: a theory of planned behavior," in Action Control: From Cognition to Behavior, J. Kuhl and J. Beckmann, Eds., SSSP Springer Series in Social Psychology, pp. 11-39, Springer, Berlin, Germany, 1985.

[38] D. Darpy, "Consumer procrastination and purchase delay," in Proceedings of the 29th Annual Conference of the European Marketing Academy (EMAC '00), Rotterdam, The Netherlands, May 2000.

[39] S. G. Moore and G. J. Fitzsimons, "Yes, we have no bananas: consumer responses to restoration of freedom," Journal of Consumer Psychology, vol. 24, no. 4, pp. 541-548, 2014.

[40] G. Pizzi and D. Scarpi, "When out-of-stock products DO backfire: managing disclosure time and justification wording," Journal of Retailing, vol. 89, no. 3, pp. 352-359, 2013.

[41] X. P. Li and Y. R. Chen, "Impacts of supply disruptions and customer differentiation on a partial-backordering inventory system," Simulation Modelling Practice and Theory, vol. 18, no. 5, pp. 547-557, 2010.

[42] S. M. Nowlis, N. Mandel, and D. B. McCabe, "The effect of a delay between choice and consumption on consumption enjoyment," Journal of Consumer Research, vol. 31, no. 3, pp. 502-510, 2004.

[43] S. Netessine and N. Rudi, "Centralized and competitive inventory models with demand substitution," Operations Research, vol. 51, no. 2, pp. 329-335, 2003.

[44] D. Huang, H. Zhou, and Q.-H. Zhao, "A competitive multipleproduct newsboy problem with partial product substitution," Omega, vol. 39, no. 3, pp. 302-312, 2011.

[45] B. O. Shubert, "A sequential method seeking the global maximum of a function," SIAM Journal on Numerical Analysis, vol. 9, no. 3, pp. 379-388, 1972.

[46] D. R. Jones, C. D. Perttunen, and B. E. Stuckman, "Lipschitzian optimization without the Lipschitz constant," Journal of Optimization Theory and Applications, vol. 79, no. 1, pp. 157-181, 1993.

[47] M. Björkman and K. Holmström, "Global optimization using the DIRECT algorithm in Matlab," Applied Optimization and Modeling, vol. 1, no. 2, pp. 17-37, 1999.

[48] R.-Q. Zhang, "A note on the deterministic EPQ with partial backordering," Omega, vol. 37, no. 5, pp. 1036-1038, 2009.

[49] R.-Q. Zhang, I. Kaku, and Y.-Y. Xiao, "Deterministic EOQ with partial backordering and correlated demand caused by crossselling," European Journal of Operational Research, vol. 210, no. 3, pp. 537-551, 2011.

[50] J. H. Mathews and K. D. Fink, Numerical Methods Using MATLAB, Prentice Hall, Upper Saddle River, NJ, USA, 2003. 


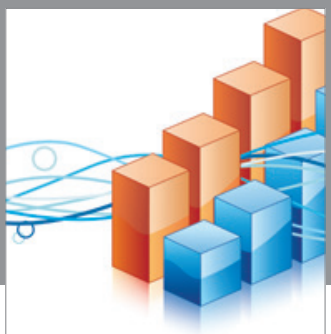

Advances in

Operations Research

vatem alat4

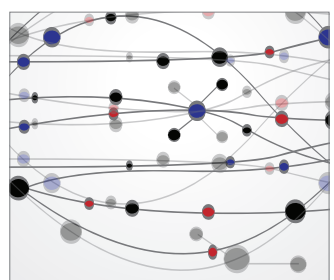

\section{The Scientific} World Journal
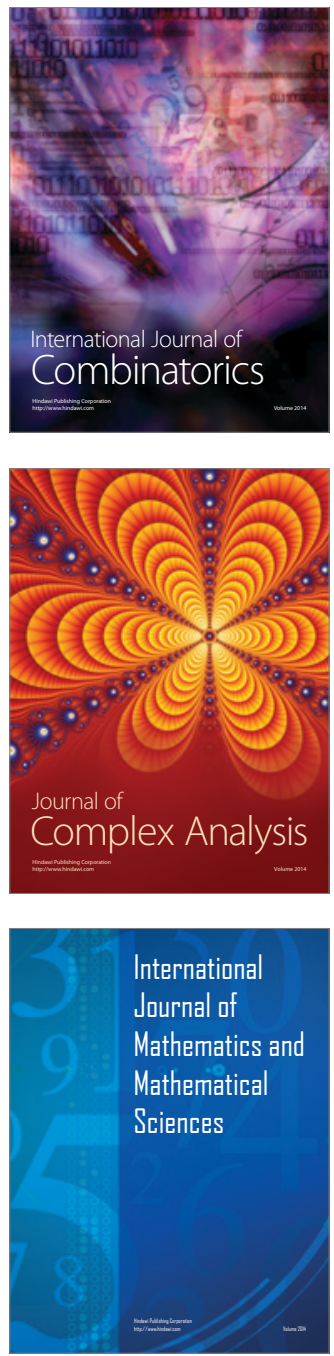
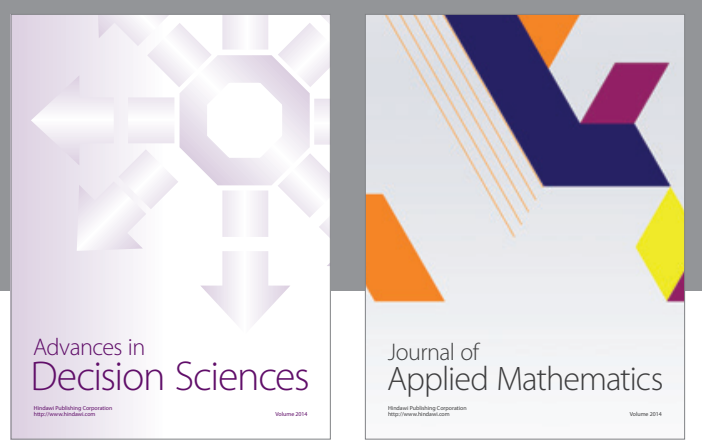

Algebra

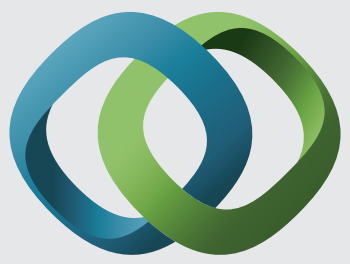

\section{Hindawi}

Submit your manuscripts at

http://www.hindawi.com
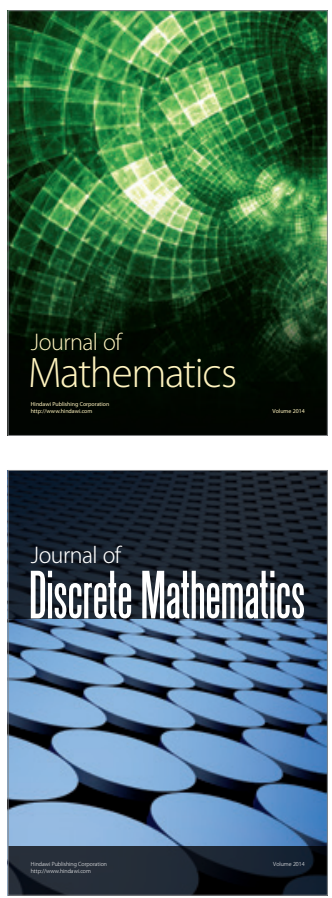

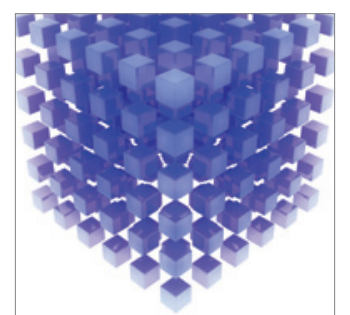

Mathematical Problems in Engineering
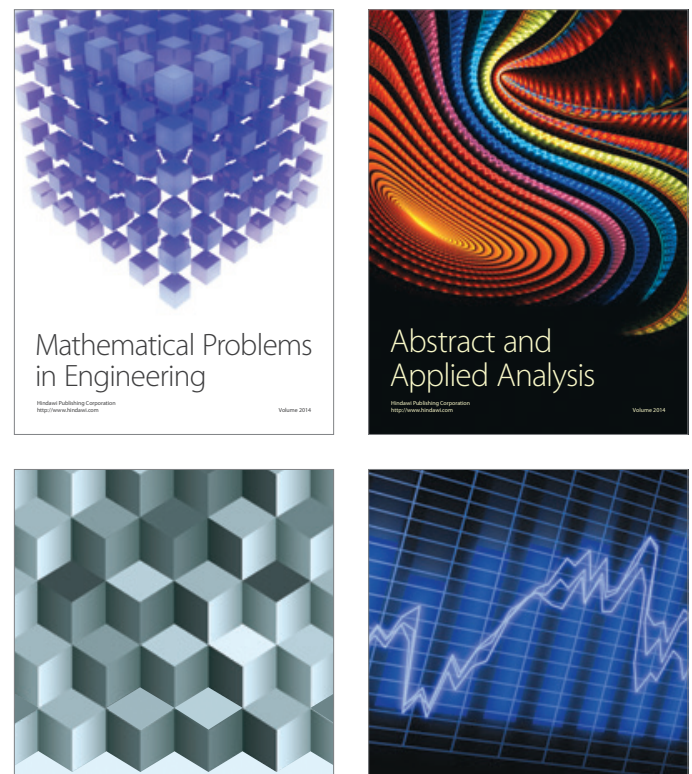

Journal of

Function Spaces

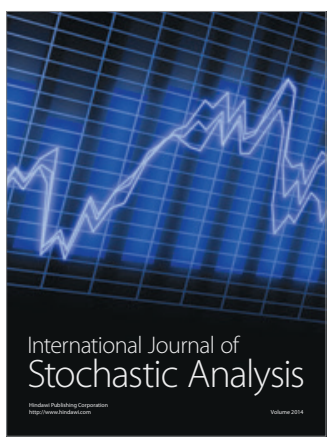

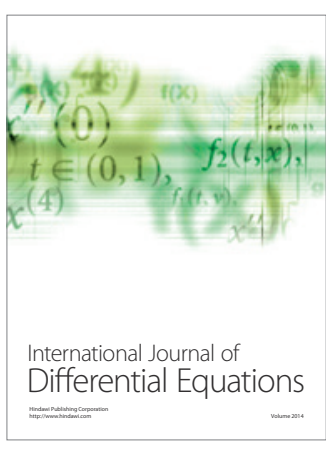
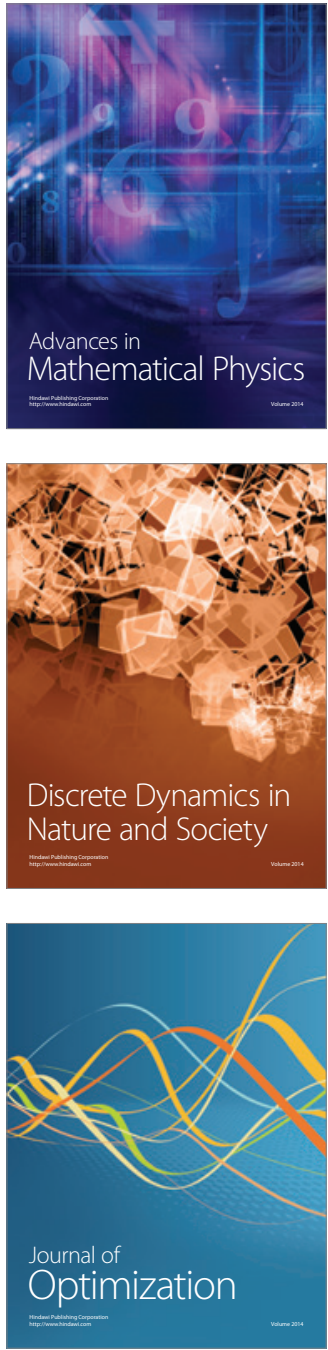